\title{
Link Quality Improvement of Long-Term Evolution and 802.11ax in High-Density Areas
}

\author{
Mina Malekzadeh (D) \\ Electrical and Computer Engineering Faculty, Hakim Sabzevari University, Sabzevar, Iran \\ Correspondence should be addressed to Mina Malekzadeh; m.malekzadeh@hsu.ac.ir
}

Received 1 April 2020; Revised 25 August 2020; Accepted 31 August 2020; Published 15 September 2020

Academic Editor: Francisco J. Martinez

Copyright (C) 2020 Mina Malekzadeh. This is an open access article distributed under the Creative Commons Attribution License, which permits unrestricted use, distribution, and reproduction in any medium, provided the original work is properly cited.

\begin{abstract}
The 802.11ax high-efficiency wireless (HEW) particularly designed for high-density areas. However, dense areas have specific requirements that demand precise deployment strategies by network developers. In dense networks, a large number of users are simultaneously connected to the same channel; hence, the available bandwidth is divided among the users in such a way that joining more users can eventually saturate the network. Furthermore, in dense areas, a large number of closely spaced users are transmitting data at the same time. In such a heavily frequency interfered environment, the wireless link quality extremely degrades, which can practically render the network unavailable. Thereby, it is essential to determine the appropriate deployment options regarding the specific networks' settings and configurations. Hence, this work proposes a network architecture model to determine the dual-band HEW performance in dense deployments. The model additionally includes long-term evolution (LTE) as the cellular alternative for high-density areas which is utilized by the model as the reference point for corresponding comparison purposes with HEW. The model is implemented, and link quality parameters are measured based on different aspects of the deployment options. To further validate the model and determine the optimization levels provided by the options, the simulation and analytical results are compared.
\end{abstract}

\section{Introduction}

The explosive growth of mobile broadband traffic is one of the current major challenges in the mobile industry. To address the capacity demands, high-efficiency wireless (HEW) 802.11ax was designed particularly for dense areas [1]. According to Cisco [2], a location is classified as high density if more than 30 active users are connected simultaneously to a single access point. However, network development in high-density areas with specific requirements is another challenge. Considering high-density environments such as airports, stadiums, concert halls, and convention centers, many users are sharing the same connection and transmitting data at the same time. This can bring any underlying network to its lowest level of performance if it is not deployed correctly. The issue essentially requires a solution regarding the ever-increasing demand for network capacity in a costefficient manner. A promising solution for this issue is to choose the appropriate deployment options and configurations regarding the network's parameters.
To succeed in the enterprise, service providers and developers need to know the optimal values of the deployment options beforehand to ensure end users' satisfaction in a cost-efficient manner. However, despite the importance, there is no current work to address the limitations and implementation issues of dual-band HEW in dense areas through the appropriate selection of the deployment options identifying which is our first motivation. Moreover, the LTE network is a well-known cellular alternative for dense areas. In this regard, our second motivation is to implement the LTE network with similar deployment options as the HEW to be able to leverage the results for practical functionality assessment of the HEW in dense areas with reference to the cellular LTE.

Accordingly, three main contributions of this work are summarized as follows. First, a network architecture model is proposed to develop three high-density networks, including LTE, HEW in $2.4 \mathrm{GHz}$ that we refer to it as HEW-2.4, and HEW in $5 \mathrm{GHz}$ that we refer to it as HEW-5. Further on this, the model takes into account six distinct deployment options each reflecting a different network aspect. The 
options include operating channel management, connection limit, antenna configuration, bandwidth requirements, Internet protocol efficiency, and payload size distribution. In this regard, determining the optimal values of the deployment options to further enhance the link quality and network capacity of HEW compared to LTE in dense areas is the second contribution of this work. Knowing the optimal values in advance before utilizing them in the dense networks will improve the end users' satisfaction and yet, maximize cost efficiency for the service providers and developers. Moreover, since determining the validity of the results is a part of any model development process, the third contribution of this work is to establish a baseline by calculating the analytical results to be compared with those of the simulation results.

The rest of the work is organized as follows. Section 2 presents related works. Section 3 provides details regarding the architecture of the model and its built-in networks, scenarios, performance metrics, and methodology followed to implement the scenarios and measure the results. Section 4 presents the results, and Section 5 concludes the work.

\section{Related Works}

As a newly released standard, there are only a few studies on HEW efficiency and functionally in conjunction with network capacity, and to the best of our knowledge, there is no comparable work with LTE in this regard. Thus, we classify the related works into the deployment of (i) LTE and (ii) HEW dual band separately. By varying the users' density, the authors in [3] examine the strength and weaknesses of transport layer protocols used for video streaming. The simulation results show the protocols execute differently on LTE networks as they depend on the network scenarios and parameter settings. While the work does not take into account the other deployment options mentioned in the earlier section, no evaluation over the HEW standard is performed. The effectiveness of the transport layer protocols is also addressed in [4] through the coexistence of LTE and Wi-Fi. The LTE is implemented in both UM and AM RLC modes in conjunction with different transport layer protocols. However, there are drawbacks to the work as it does not examine other deployment options and also, it does not implement HEW. By contrast, in addition to the effectiveness of the transport layer, we take into account all important deployment options between LTE and HEW to accomplish a comprehensive comparison of their performance. The coexistence of HEW and LTE networks is also provided in [5] to evaluate the signal to noise ratio level. Traffic pattern in terms of packet size and the corresponding delay imposed on LTE networks is investigated in [6]. Through the measurement results, they illustrate that shorter packets are more suitable for delay-sensitive applications as they improve the end-to-end delay performance of LTE users.

However, other performance metrics, deployment options, and HEW networks are not evaluated. On the contrary, we take into account all the common deployment options of both LTE and HEW to be as comprehensive as possible. A testbed is set up in [7] to characterize the performance of IPv6 and compare it with IPv4 in only LTE net- works. The average time for establishing a TCP connection for different websites is measured from IPv6 to IPv6, IPv6 to $\mathrm{IPv} 4$, and $\mathrm{IPv} 4$ to IPv4. The results indicate a longer connection time for IPv6 than IPv4. Other than comparing IPv6 and IPv4, the experiments are limited to the LTE network, and they do not consider HEW or any other performance metrics and deployment options. In [8], the authors use MATLAB and Vienna LTE-A system-level simulator to evaluate the performance of four LTE channel widths with MIMO options, but no evaluation is performed over HEW networks. MATLAB is also used by authors in [9] to investigate different MIMO transmission modes in LTE. Due to being a new standard, with regards to HEW, there are only a few current works with no comparison over LTE. The user density in HEW is investigated in [10] in terms of throughput and latency. A testbed is set up in [11] to compare throughput and round-trip time of HEW with standard datasets that exist for IEEE $802.11 \mathrm{~g}$ networks. The authors in [12] perform analysis for different node densities and traffic types in HEW network. In [13], the authors suggest a TCP-aware scheduling strategy for optimizing the transmission of TCP traffic. The number of stations and resource allocation algorithms are the only deployment options evaluated in [14, 15], respectively, to measure the corresponding effects. However, no investigation of other critical deployment options or any comparison with LTE network is provided.

Despite the existing works, two main limitations are identified. First, there is a lack of enough information regarding the practical functionality of the HEW in high-density areas with regard to the effective deployment options. Second, while both LTE and HEW have been developed with supporting high-density areas in mind, no comparable work between them exists to evaluate their contribution to the networks' performance optimization. This work proposes a model to address both limitations in high-density deployments. The model provides an in-depth evaluation of the common deployment options between HEW and LTE networks in high dense areas. This further determines the actual performance gains and optimal values in advance before applying the options on the networks, which in turn allows the developer and service providers to meet the increasing demands of network capacity.

\section{Model Implementation}

The procedure followed in this work starts with developing a model to address the capacity issues in dense HEW and LTE networks concerning the efficiency of the deployment options and to measure the collective effects in both. The optimal values of the options are determined to achieve the best possible results in high dense HEW and LTE networks. The architecture of the model, the scenarios designed in the network simulator, and the methodology followed to implement the scenarios and measure the results in terms of link quality performance metrics are discussed in detail in this section.

3.1. Network Architecture. The model primarily includes three distinct network architectures, including LTE, HEW in $2.4 \mathrm{GHz}$, and $\mathrm{HEW}$ in $5 \mathrm{GHz}$ frequency bands as follows. 
3.1.1. LTE Network Architecture. The LTE network developed in the model is designed with full evolved packet core (EPC) architecture. The user equipment (UEs) are connected to eNodeB through which the data packets are transmitted in the downlink direction from the remote server toward the UEs. The eNodeB accomplishes this via connection to the EPC core nodes, including serving gateway (SGW) and packet data network gateway (PGW) in the presence of a mobility management entity (MME). The UEs are initially positioned randomly around a rectangular grid and then move in random directions in the closed area of the grid. The direction boundary (meter), speed (meters per second), and pause time and delay (second) mobility parameters for each UE are controlled by rectangle (rectangle $(-100 \mathrm{~m}$, $100 \mathrm{~m},-100 \mathrm{~m}, 100 \mathrm{~m})$ ), constant random (constant random variable $[$ constant $=0.2]$ ), and uniform random (uniform random variable $[\operatorname{Min}=1 \mid \operatorname{Max}=6]$ ), respectively. The $\mathrm{UE}$ pauses for the specified delay to choose a random direction and speed in the range of 1 to $6 \mathrm{~m} / \mathrm{s}$. Then, the UE travels in the selected direction with the selected speed until it reaches the predefined rectangle boundary. After that, it pauses again and selects a new direction and speed. This process repeats until the end of the simulation time (200 seconds) to represent real-world mobility in a closed area. In order to allocate resources to UEs, the proportional fair scheduling algorithm is used. Figure 1 shows the graphical representations for 100 UEs in the LTE network before and during data reception to visualize the cell signal coverage by the eNodeB.

3.1.2. HEW-2.4 and HEW-5 Networks Architecture. Since HEW is dual band, the model includes two distinct HEW networks, one in the $2.4 \mathrm{GHz}$ frequency band, which is for simplicity, we refer to it as HEW-2.4, and the other in the $5 \mathrm{GHz}$ frequency band which we refer to it as HEW-5. For comparison purposes, the architecture and corresponding settings and parameters of the HEW-2.4 and HEW-5 first must be identical (except for the operational frequency band) and second, be comparable to those for the LTE network in the model. This is essential for providing the highest possible level of fair conditions for comparison purposes between LTE and HEW networks. The HEW stations (STAs) are randomly positioned with similar mobility parameters as the LTE network. The STAs are connected to the HEWenabled access point, which is responsible for data delivery in the downlink direction from the remote server to the connected STAs. Moreover, for sending more data in a single transmission, the default frame aggregation method called aggregated-MAC packet data unit (A-MPDU) is used in both HEW networks. Figure 2 indicates the HEW-5 network in the model for 100 STAs.

3.2. Simulation Scenarios. The three developed networks in the model are used as underlying networks over which the corresponding scenarios are implemented, and the performance results are measured. Due to the structural difference of cellular LTE and wireless HEW networks, there exist multiple deployment options at different layers that can affect the overall network capacity. With both service providers and end users in mind, the model takes into account six deployment options common in HEW and LTE networks and corresponds them to the following six classes of scenarios.

3.2.1. Operating Channel Management. This class of scenarios focuses on the aspects of the available channels in LTE, HEW-2.4, and HEW-5 networks to identify their efficiency and thereby determine the optimal selections under highdensity conditions. The details are as follows.

(1) Operating Channel Management for the LTE Network in the Model. The LTE frequency range is $600 \mathrm{MHz}$ to $6 \mathrm{GHz}$, and the available channel widths are $1.4,3,5,10,15$, and 20 [16]. Therefore, there are multiple operating channel bands for LTE, while each band supports a range of frequencies [17]. Among the existing bands, we select band seven with $2.655 \mathrm{GHz}$ center frequency. The reason behind this selection, apart from being close to the frequency of HEW-2.4 network, is that band seven is among the most common internationally used bands [18]. It has high data capacity and can deal with the high number of devices connecting at once, making it ideal for dense areas [19]. For this band, the possible channel widths are $5,10,15$, and $20 \mathrm{MHz}$, which corresponds to 25 , 50,75 , and 100 resource blocks (RBs), respectively [20,21]. For this class of scenarios, the LTE network supports and implements all four channel widths while for other classes, the default value $(20 \mathrm{MHz})$ is implemented. The parameters related to the LTE band seven used by the model along with the absolute radio frequency channel number (EARFCN) are presented in Table 1.

(2) Operating Channel Management for the HEW-2.4 Network in the Model. The $2.4 \mathrm{GHz}$ frequency spectrum typically consists of eleven channels (1 to 11). Among the nonoverlapping channels $(1,6$, and 11$)$, we select the first channel for the HEW-2.4 in the model which supports four different channel widths of $20,40,80$, and $160 \mathrm{MHz}$. Therefore, this class of scenarios also takes into account the implementation of all four channel widths while for other classes, the default value $(40 \mathrm{MHz})$ is used. The simulation parameters of the HEW2.4 network for channel one are presented in Table 2.

(3) Operating Channel Management for the HEW-5 Network in the Model. In the $5 \mathrm{GHz}$ frequency band, the HEW standard has channel numbers ranging from 36 up to 165 each supporting a different channel width [22]. Hence, to support all the four channel widths by the HEW-5, the selected channel numbers are $36,38,42$, and 50 correspond to $20,40,80$, and $160 \mathrm{MHz}$ channel widths, respectively. Moreover, for other classes of scenarios, the default channel width ( $40 \mathrm{MHz}$ ) is used. Thus, the model for the HEW-5 network considers all four channel widths with the first channel numbers as described in Table 3.

3.2.2. Maximum Connection Limit. In order to ensure reliability and stability, identifying the maximum connection limit is one of the essential options that must be determined before the deployment of the networks. Accordingly, in this class of scenarios, the overall performance of the LTE, 


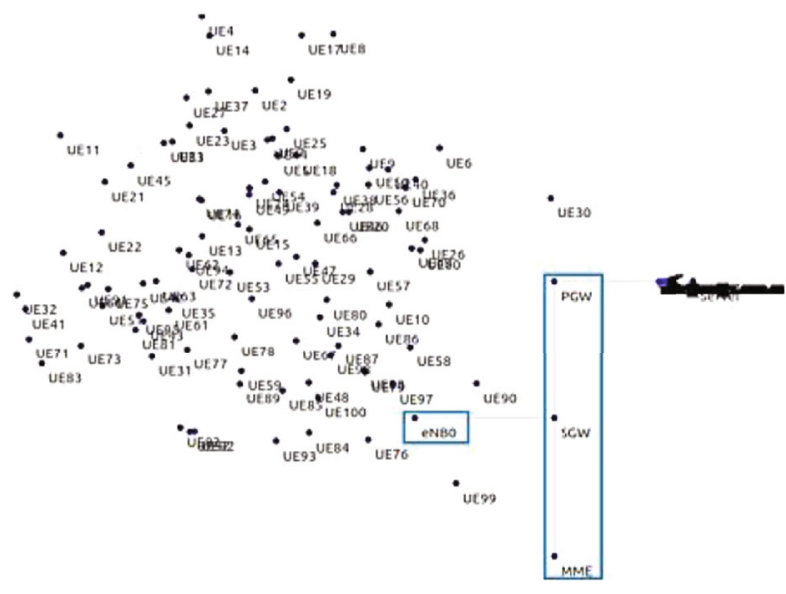

(a)

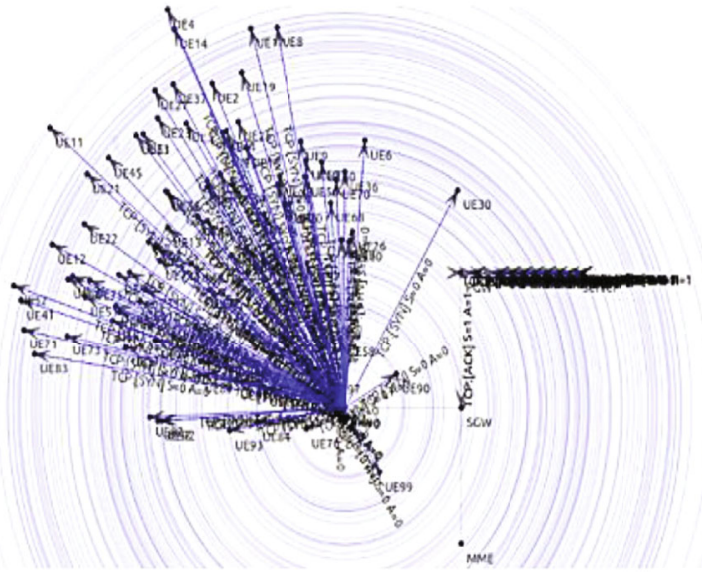

(b)

FIGURE 1: The graphical representation for 100 UEs in LTE network: (a) before and (b) during data reception.

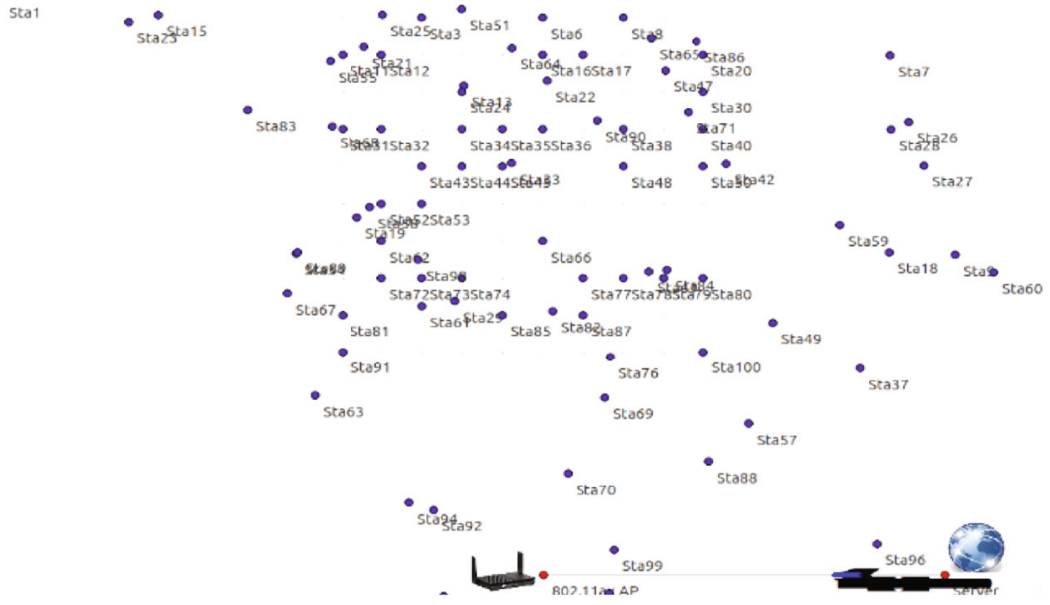

FIgURE 2: The graphical representation for 100 STAs in the HEW-5 network.

TABLE 1: Simulation parameters related to the LTE channel.

\begin{tabular}{lc}
\hline Parameters & Description \\
\hline Channel number & 7 \\
Channel name & 2600 \\
Center frequency and EARFCN & 2655 and $3100 \mathrm{MHz}$ \\
Lower frequency and EARFCN & 2620 and $2750 \mathrm{MHz}$ \\
Higher frequency and EARFCN & 2690 and $3449 \mathrm{MHz}$ \\
Channel width support & $5,10,15,20 \mathrm{MHz}$ \\
\hline
\end{tabular}

TABLE 2: Simulation parameters related to the HEW-2.4 channel.

\begin{tabular}{lc}
\hline Parameters & Description \\
\hline Channel number & 1 \\
Center frequency & $2412 \mathrm{MHz}$ \\
Lower frequency & $2402 \mathrm{MHz}$ \\
Higher frequency & $2422 \mathrm{MHz}$ \\
Channel width & $20,40,80,160 \mathrm{MHz}$ \\
\hline
\end{tabular}

HEW-2.4, and HEW-5 networks with different densities of users are measured and evaluated. By aiming to determine any possible scalability limit in LTE, HEW-2.4, and HEW-5 networks and evaluate how these networks can tolerate the increasing number of active users, we vary the density of the users in the range of $10,30,50,70,90,110,130$, and 150 users. Further on this, for other classes of scenarios, 100 active users are connected in each network. The reason is related to the $20 \mathrm{MHz}$ channel width in LTE which includes $100 \mathrm{RBs}$ in total in each transmission time interval (TTI) for all the users.

3.2.3. Antenna Configuration. This class of scenarios mainly attempts to identify that utilizing which antenna configuration technique leads to performance optimization of high dense HEW-2.4 and HEW-5 compared to LTE networks. The details are as follows.

(1) Antenna Configuration for the LTE Network in the Model. With regards to the antenna's configuration for data transmission, the three following modes are supported by the model: 
TABLe 3: Simulation parameters related to the HEW-5 channel.

\begin{tabular}{lcccc}
\hline $\begin{array}{l}\text { Channel } \\
\text { number }\end{array}$ & $\begin{array}{c}\text { Channel } \\
\text { width }\end{array}$ & $\begin{array}{c}\text { Center } \\
\text { frequency }\end{array}$ & $\begin{array}{c}\text { Lower } \\
\text { frequency }\end{array}$ & $\begin{array}{c}\text { Higher } \\
\text { frequency }\end{array}$ \\
\hline 36 & $20 \mathrm{MHz}$ & $5180 \mathrm{MHz}$ & $5170 \mathrm{MHz}$ & $5190 \mathrm{MHz}$ \\
38 & $40 \mathrm{MHz}$ & $5190 \mathrm{MHz}$ & $5170 \mathrm{MHz}$ & $5210 \mathrm{MHz}$ \\
42 & $80 \mathrm{MHz}$ & $5210 \mathrm{MHz}$ & $5170 \mathrm{MHz}$ & $5250 \mathrm{MHz}$ \\
50 & $160 \mathrm{MHz}$ & $5250 \mathrm{MHz}$ & $5170 \mathrm{MHz}$ & $5330 \mathrm{MHz}$ \\
\hline
\end{tabular}

Mode 1: this mode is the default mode and considers a single antenna for eNodeB

Mode 2: this mode is the transmit diversity and for this purpose, it considers two antennas for transmission of two copies of the same data [23]

Mode 3: this mode is called open-loop spatial multiplexing (OLSM). It also considers two antennas but for the transmission of two separate data streams $[24,25]$

(2) Antenna Configuration for HEW-2.4 and HEW-5 Networks in the Model. The antenna configuration for both HEW-2.4 and HEW-5 is represented as $N_{\mathrm{Tx}} \times N_{\mathrm{Rx}}: N_{\mathrm{SS}}$, where $N_{\mathrm{Tx}}, N_{\mathrm{Rx}}$, and $N_{\mathrm{SS}}$ are the number of transmitting antenna, receiving antenna, and spatial streams, respectively [26]. The model for both HEW-2.4 and HEW-5 supports four modes so that the number of $N_{\mathrm{Tx}}, N_{\mathrm{Rx}}$, and $N_{\mathrm{SS}}$ is equal as follows:

Mode 1: this default mode considers one single antenna for both transmitter and receiver with one special stream $(1 \times 1: 1)$.

Mode 2: this mode considers that the transmitter and receiver have two antennas for exchanging two spatial streams $(2 \times 2: 2)$.

Mode 3: in this mode, three antennas are defined for transmitter and receiver for exchanging three spatial streams $(3 \times 3: 3)$.

Mode 4: this mode increases the number of transmitter's and receiver's antennas to four to exchange four special streams $(4 \times 4: 4)$.

For other classes of scenarios, the mode 1 is the default mode for LTE and HEW networks. It is also worth noting that the HEW standard can use orthogonal frequency division multiple access (OFDMA) which is a multiuser version of OFDM. However, since the network simulator does not support multiuser mode, the model includes OFDM for both HEW networks.

3.2.4. Bandwidth Requirements. In high-density environments such as campuses and large events spaces such as stadiums, concert halls, and convention centers, users exchange different types of data from low-bandwidth applications such as simple web surfing to high-bandwidth applications such as video streaming. Some of these applications running on the network are error-sensitive and some are time-sensitive. From the time standpoint, the real challenges with timesensitive applications are jitter and having zero tolerance for delay [27]. Thus, they are commonly transmitted using the user datagram protocol (UDP) to meet their real-time needs [28]. On the contrary, the major problem of errorsensitive applications is accuracy failure by occurring packet loss due to errors or excessive delay. Thus, they carry data using the transmission control protocol (TCP), which, as a reliable protocol, ensures involving error control mechanisms such as retransmission [29, 30]. Accordingly, to facilitate proper bandwidth utilization in conjunction with bandwidth requirements of the applications, the model supports both error-sensitive and time-sensitive applications in LTE, HEW-2.4, and HEW-5 networks under high-density conditions.

3.2.5. Internet Protocol (IP) Efficiency. The limited address capacity of IPv4 imposes severe constraints on the development of the dense networks. The highly scalable address scheme of IPv6 solves this issue for the ever-increasing number of connected devices. The fact is that organizations and service developers will eventually need to run IPv6only on their systems; hence, a transition to IPv6 is unavoidable. However, despite its benefits, there are critical ambiguities and concerns about the practical IPv6 performance and reliability for adaption in high-density mobile networks. Therefore, it is highly important to establish a baseline for clarifying any network performance benefit offered by IPv6 to practically justify its deployment by service providers and developers. With this in mind, the aim in this class of scenarios is to adopt IPv6-only and IPv4only in highly dense LTE, HEW-2.4, and HEW-5 networks and verify any possible outperformance of IPv6 and IPv4 over each other. For other classes of scenarios, IPv6 is the default protocol.

3.2.6. Payload Size Distribution. This class of scenarios attempts to determine the optimal size for efficient data transmission in high dense LTE, HEW-2.4, and HEW-5 networks. For this purpose, the model includes applications for generating three distinct payload sizes as 700, 1400, and 2800 bytes with respect to $1460 \mathrm{~B}$ as the maximum transmission unit (MTU) [31-33]. This can determine whether the segmentation, fragmentations, and the other overheads caused by packets with a size smaller or larger than MTU impose bottleneck to the high-density LTE, HEW-2.4, or HEW-5 networks, or they can efficiently handle the overheads. This can further identify the optimal payload size for different network applications. For other classes of scenarios, $1400 \mathrm{~B}$ is the default size.

It is worth noting that for all the six classes of scenarios in the model, we consider 200 replicates for each scenario. The above parameters in the context of the scenarios are summarized in Table 4.

3.3. Link Quality Evaluation Metrics. All the designed scenarios are implemented separately over the three developed networks LTE, HEW-2.4, and HEW-5. The results are measured in terms of link quality metrics to characterize the performance in high-density areas and identify the optimal values. There are two types of link quality parameters: hardware- 
TABLE 4: Simulation parameters.

\begin{tabular}{lc}
\hline Options and parameters & Values \\
\hline Channel width & LTE: $5,10,15,20 \mathrm{MHz}$ \\
User density & HEW: 20, 40, 80,160 MHz \\
Antenna configuration & LTE: mode 1 to 3 \\
Internet protocol & HEW: mode 1 to 4 \\
Transport payload size & IPv6, IPv4 \\
Traffic type & 700, 1400, 2800 bytes \\
Traffic pattern & Error-sensitive (TCP), \\
Traffic generation rate & delay-sensitive (UDP) \\
Mobility & Constant bit rate (CBR) \\
& 2 Mbps \\
\hline
\end{tabular}

based and software-based [34]. The software-based link quality (SBLQ) parameters are measured at the receiver side based on statistics of the received packets. The SBLQ parameters supported by the model include throughput, end-toend delay, packet loss ratio, and jitter. Moreover, starvation is a well-known issue caused by channel access competition of a large number of users in high dense networks which results in certain users wait for a longer time than the others [26]. To determine how HEW and LTE can manage the starvation in dense conditions, the fairness index (FI) measurement is provided by the model for the scenarios that vary the density of the users (maximum connection limit class). The fairness index represents the equality of allocating the resources to individual users by the underlying network. It ranges from 0 to 1 , with 1 showing $100 \%$ network fairness so that all the users receive a completely fair share of the desired resources [34]. To determine the throughput fairness provided for each user by the LTE, HEW-2.4, and HEW-5 networks under high-density conditions, the fairness index, FI, is calculated as

$$
\mathrm{FI}=\frac{\left(\mathrm{ST}_{1}+\mathrm{ST}_{2}+\cdots+\mathrm{ST}_{N}\right)^{2}}{N \times\left(\mathrm{ST}_{1}^{2}+\mathrm{ST}_{2}^{2}+\cdots+\mathrm{ST}_{N}^{2}\right)} \times 100
$$

where $\mathrm{ST}_{i}$ is the simulation throughput of the $i^{\text {th }}$ user, $N$ is the total number of users, and $i$ is considered as $1 \leq i \leq N$; $N \in\{10,30,50,70,90,110,130,150\}$.

In contrast, the hardware-based link quality (HBLQ) parameters are acquired directly from the radio transceivers. In this regard, the model includes received signal strength indicator (RSSI), signal-to-noise ratio (SNR), reference signal received power (RSRP), reference signal received quality (RSRQ), and channel capacity (CC) [35, 36]. Two distinct modules are written for the HEW-2.4 and HEW-5 networks to measure SNR and RSSI parameters of the STAs. Similarly, two distinct modules are written for the LTE network to measure SNR and RSRP
TABLE 5: LTE RSSI and RSRQ measurements in the model.

\begin{tabular}{lccc}
\hline Channel width & $N_{\mathrm{RB}}$ & RSSI $(\mathrm{dBm})$ & RSRQ $(\mathrm{dB})$ \\
\hline $5 \mathrm{MHz}$ & 25 & RSRP +24.77 & $13.97+$ RSRP - RSSI \\
$10 \mathrm{MHz}$ & 50 & RSRP +27.78 & $16.98+$ RSRP - RSSI \\
$15 \mathrm{MHz}$ & 75 & RSRP +29.54 & $18.75+$ RSRP - RSSI \\
$20 \mathrm{MHz}$ & 100 & RSRP +30.79 & $20+$ RSRP - RSSI \\
\hline
\end{tabular}

parameters of the UEs. On this basis, RSSI and RSRQ of the UEs are also measured as follows $[16,36]$ :

$$
\begin{aligned}
\mathrm{RSSI} & =\mathrm{RSRP}+10 \times \log _{10}\left(12 \times N_{\mathrm{RB}}\right), \\
\mathrm{RSRQ} & =10 \times \log _{10}\left(N_{\mathrm{RB}}\right)+\mathrm{RSRP}-\mathrm{RSSI},
\end{aligned}
$$

where $N_{\mathrm{RB}}$ is the number of resource blocks.

The summary of the RSSI and RSRQ measurements for the LTE network is provided in Table 5.

For LTE, HEW-2.4, and HEW-5 networks, the channel capacity (CC) represents the maximum rate at which data can be reliably transmitted over a given channel and is calculated in bps based on the Shannon-Hartley formula [37-39] as follows:

$$
\mathrm{CC}=\mathrm{CW} \times \log _{2}\left(1+N_{\mathrm{Tx}} \times N_{\mathrm{Rx}} \times \mathrm{SNR}_{\mathrm{avg}}\right),
$$

where CC is the channel capacity, CW is the channel width, $N_{\mathrm{Tx}}$ is the number of transmitting antenna, $N_{\mathrm{Rx}}$ is the number of receiving antenna, and $\mathrm{SNR}_{\mathrm{avg}}$ is the average SNR of the channel.

3.4. Validity Assessment of the Model. Determining the validity of the results is a part of any model development process to guarantee the integrity of any further decision-making approaches based on those results. In this context, as the verification strategy for establishing reliability and validity of the measured simulation results, we compare them with the results obtained with the aid of analytical calculations. For this purpose, the model further takes into consideration the analytical metrics including the maximum achievable throughput (MAT), efficiency ratio (ER), and peak throughput in the physical layer $\left(\mathrm{PT}_{\mathrm{PL}}\right)$. In this regard, the notations used throughout this work are introduced in Table 6.

3.4.1. Maximum Achievable Throughput. The maximum achievable throughput (MAT) determines the average maximum possible quantity of data that is received by the users per given time [26]. First, we measure the simulation MAT $\left(\mathrm{MAT}_{\mathrm{S}}\right)$, and then we calculate the analytical MAT $\left(\mathrm{MAT}_{\mathrm{A}}\right)$. The aim is to compare the $\mathrm{MAT}_{S}$ results with analytical $\mathrm{MAT}_{\mathrm{A}}$ to determine the validity of the simulation results. $\mathrm{MAT}_{\mathrm{A}}$ is a function of several parameters and is calculated as

$$
\mathrm{MAT}_{\mathrm{A}}=\frac{N_{\mathrm{DS}} \times N_{\mathrm{BPS}} \times \mathrm{CR} \times N_{\mathrm{SS}}}{T_{\mathrm{SD}}+T_{\mathrm{GI}}},
$$

where $N_{\mathrm{DS}}$ is the number of data subcarriers, $N_{\mathrm{BPS}}$ is the 
TABLE 6: Notations and descriptions.

\begin{tabular}{|c|c|c|c|}
\hline Notation & Description & Notation & Description \\
\hline $\mathrm{CP}$ & Cyclic prefix & $N_{\mathrm{RE}}$ & Number of resource elements \\
\hline CW & Channel width (MHz) & $N_{\text {S_OFDM }}$ & Number of OFDM symbols \\
\hline $\mathrm{CR}$ & Coding rate & $N_{\text {SC_RB }}$ & Number of subcarriers per resource block \\
\hline $\mathrm{ER}_{\mathrm{AS}}$ & Analytical and simulation efficiency ratio & $N_{\text {SL_SF }}$ & Number of slots per sub-frame \\
\hline$L_{\mathrm{CRC}}$ & Length of cyclic redundancy check (bits) & $N_{\text {SS }}$ & Number of spatial streams \\
\hline $\mathrm{MAT}_{\mathrm{A}}$ & Analytical maximum achievable throughput & $N_{\mathrm{SSF}}$ & Number of scheduled sub-frames \\
\hline $\mathrm{MAT}_{\mathrm{S}}$ & Simulation maximum achievable throughput & $P_{\mathrm{RE}}$ & Percentage of RE in the control channel \\
\hline MCS & Modulation coding scheme & $\mathrm{PT}_{\mathrm{PL}}$ & Peak throughput in the physical layer \\
\hline$N_{\text {BPS }}$ & Number of bits per symbol & $T_{\mathrm{GI}}$ & Guard interval time $(\mu \mathrm{s})$ \\
\hline$N_{\mathrm{DS}}$ & Number of data subcarriers & $T_{\mathrm{SD}}$ & Symbol duration time $(\mu \mathrm{s})$ \\
\hline$N_{\mathrm{RB}}$ & Number of resource blocks & TBS & Transport block size (bit) \\
\hline
\end{tabular}

number of bits per symbol, CR is the coding rate, $N_{\mathrm{SS}}$ is the number of special streams, $T_{\mathrm{SD}}$ is the symbol duration, and $T_{\mathrm{GI}}$ is the guard interval time. Due to structural differences between LTE and HEW networks, each parameter in equation (4) for LTE is different than HEW; thus, we need to consider them separately as follows.

(1) MATA Calculation in HEW. The $N_{\text {DS }}$ in HEW networks depends on the CW. Accordingly, the $N_{\text {DS }}$ values for 20, 40, 80 , and $160 \mathrm{MHz}$ channels are 234, 468, 980, and 1960, respectively [40]. Unlike LTE, the modulation coding scheme (MCS) can be adjusted in HEW networks. While the possible MCS values in the HEW networks are from 0 to 11 , the default MCS in the model is 8 which results in utilizing the 256-QAM algorithm for modulation [40]. This in turn provides the $N_{\text {BPS }}$ as 8 bits and $C R$ as 3/4 [40]. Moreover, as mentioned earlier, the $N_{\mathrm{SS}}$ values for mode 1 to 4 in HEW networks are 1 to 4 , respectively. While $T_{\mathrm{SD}}$ is $12.8 \mu \mathrm{s}$, the HEW standard includes three guard interval time $\left(T_{\mathrm{GI}}\right)$ values as $0.8,1.6$, and $3.2 \mu \mathrm{s}$ [40]. On the other hand, in dense networks, a large number of closely spaced users are transmitting data at the same time resulting in such a heavily frequency interfered environment. For this reason, we select $0.8 \mu \mathrm{s}$ for $T_{\mathrm{GI}}$ to avoid more signal interference. Given all these together, the simplified form of equation (4) in Mbps for the HEW networks in the model will be as follows:

$$
\mathrm{MAT}_{\mathrm{A}}=0.441 \times N_{\mathrm{DS}} \times N_{\mathrm{SS}} .
$$

(2) MATA Calculation in LTE. In the LTE network, the $N_{\mathrm{DS}}$ values for $5,10,15$, and $20 \mathrm{MHz}$ channels are 301, 601, 901, and 1201, respectively [41]. Unlike HEW networks, the MCS in the LTE network is not adjustable and varies depending on the radio link quality and conditions. The link with better quality achieves a higher MCS which allows more data transmission. By looking at the simulation results, we determine that the achieved MCS based on the channel conditions is 28. Thus, we also consider the same value for further analytical calculations. This MCS value corresponds to the 64-QAM modulation algorithm and thereby, the $N_{\text {BPS }}$ will be 6 [41]. With regard to the $C R$ value in the LTE network, it relies on different parameters and is calculated as follows:

$$
\mathrm{CR}=\frac{\mathrm{TBS} \times L_{\mathrm{CRC}}}{N_{\mathrm{RE}} \times N_{\mathrm{BPS}}},
$$

where TBS is the transport block size, $L_{\mathrm{CRC}}$ is the length of cyclic redundancy check (CRC), $N_{\mathrm{RE}}$ is the number of resource elements, and $N_{\mathrm{BPS}}$ is the number of bits per symbol. The size of the transport block relies on the CW; thus, the TBS values for $5,10,15$, and $20 \mathrm{MHz}$ channels are $18336,36696,55056$, and 75376, respectively [41]. Moreover, the $L_{\mathrm{CRC}}$ is 24 bits in LTE networks. The $N_{\mathrm{RE}}$ as a function of different parameters is calculated as follows:

$$
N_{\mathrm{RE}}=N_{\mathrm{RB}} \times N_{\mathrm{SC} \_\mathrm{RB}} \times N_{\mathrm{S} \_\mathrm{OFDM}} \times N_{\mathrm{SL} \_\mathrm{SF}} \times\left(1-\frac{P_{\mathrm{RE}}}{100}\right) \text {, }
$$

where $N_{\mathrm{RB}}, N_{\mathrm{SC} \_\mathrm{RB}}, N_{\mathrm{S} \_ \text {OFDM }}$, and $N_{\mathrm{SL} \_\mathrm{SF}}$ are the number of resource blocks, subcarriers per resource block, OFDM symbols, and slots per subframe, respectively. The $N_{\mathrm{RB}}$ relies on the CW; thus, the values for $5,10,15$, and $20 \mathrm{MHz}$ channels are $25,50,75$, and 100 , respectively. With regard to $N_{\text {SC_RB }}$, there are 12 subcarriers in each LTE resource block. Moreover, depending on the cyclic prefix (CP) to be normal or extended, the number of OFDM symbols $\left(N_{S_{-} \text {OFDM }}\right)$ can be 7 or 6 , respectively [41]. Since the model includes the normal $\mathrm{CP}$, the $N_{\mathrm{S}_{-} \mathrm{OFDM}}$ will be 7 . Moreover, in LTE networks, each subframe is divided into two slots; hence, $N_{\mathrm{SL} \_\mathrm{SF}}$ is 2 . The $P_{\mathrm{RE}}$ is the percentage of resource elements (RE) in the control channel which usually is $10 \%$ [41]. Given all these together, the simplified form of equation (4) in Mbps for the LTE network in the model will be as follows:

$$
\operatorname{MAT}_{\mathrm{A}}(\mathrm{Mbps})=0.089 \times N_{\mathrm{DS}} \times N_{\mathrm{SS}} \times\left(\frac{\mathrm{TBS}+24}{777.6 \times N_{\mathrm{PRB}}}\right) .
$$




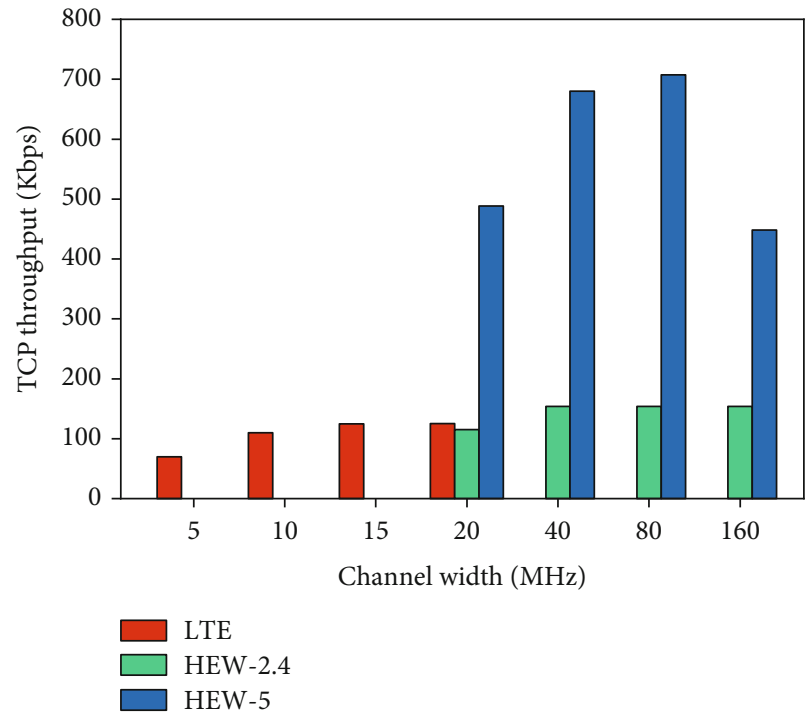

(a)

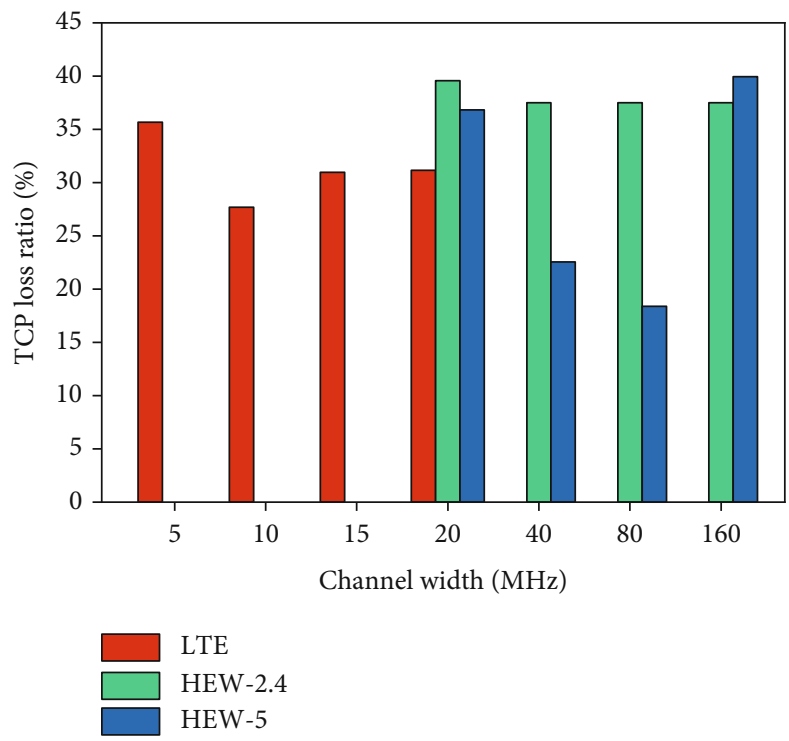

(c)

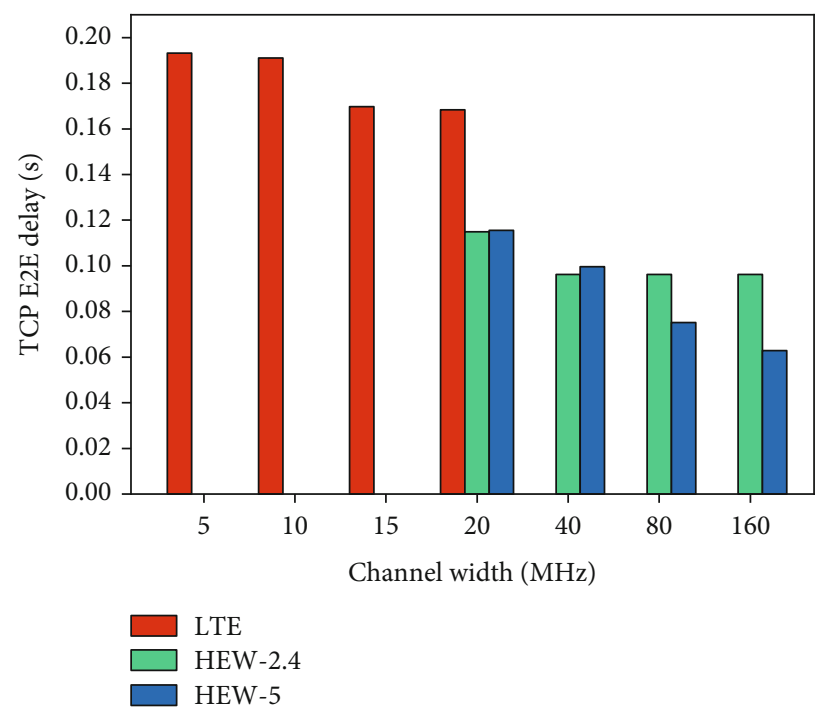

(b)

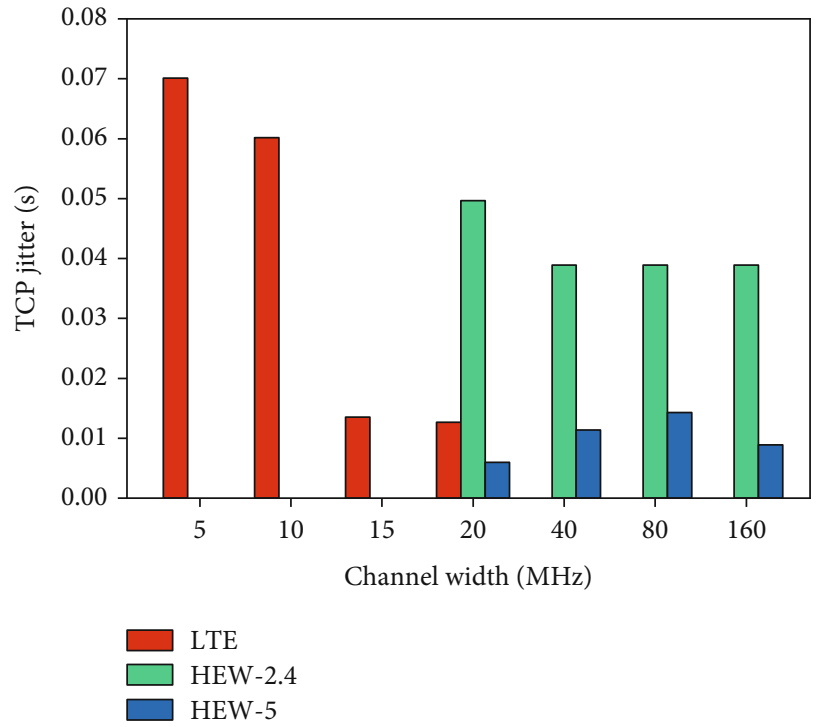

(d)

Figure 3: Operating channel width management: TCP (a) throughput, (b) delay, (c) loss ratio, and (d) jitter.

3.4.2. Efficiency Ratio of the Analytical and Simulation Results. To further determine the precision level of the simulation measurements, we calculate the efficiency ratio which represents the closeness percentage of the simulation and analytical results [35]. Thereby, we use both the analytical and simulation maximum achievable throughputs to calculate the analytical and simulation efficiency ratio $\left(E R_{A S}\right)$ as follows:

$$
\mathrm{ER}_{\mathrm{AS}}=\frac{\mathrm{MAT}_{\mathrm{A}}-\mathrm{MAT}_{\mathrm{S}}}{\mathrm{MAT}_{\mathrm{A}}} \times 100,
$$

where $\mathrm{MAT}_{\mathrm{A}}$ and $\mathrm{MAT}_{\mathrm{S}}$ are the analytical and simulation maximum achievable throughputs, respectively. The $\mathrm{ER}_{\mathrm{AS}}$ is expressed as a percentage to analyze how well the model works so that the lower the ratio, the better. A lower ratio is an indication of higher accuracy of the simulation results as their differences with the analytical results decreases. In this regard, if the efficiency ratio increases, it means the simulation results have a significant difference with the analytical results which is not desirable.

3.4.3. Peak Throughput in the Physical Layer. In LTE networks, radio frames are used to carry data between users and eNodeB while each frame contains ten subframes. In order to transmit data, it is delivered from the upper layers to the physical layer in the form of the transport block to be transmitted in $1 \mathrm{~ms}$ subframes correspond to the 


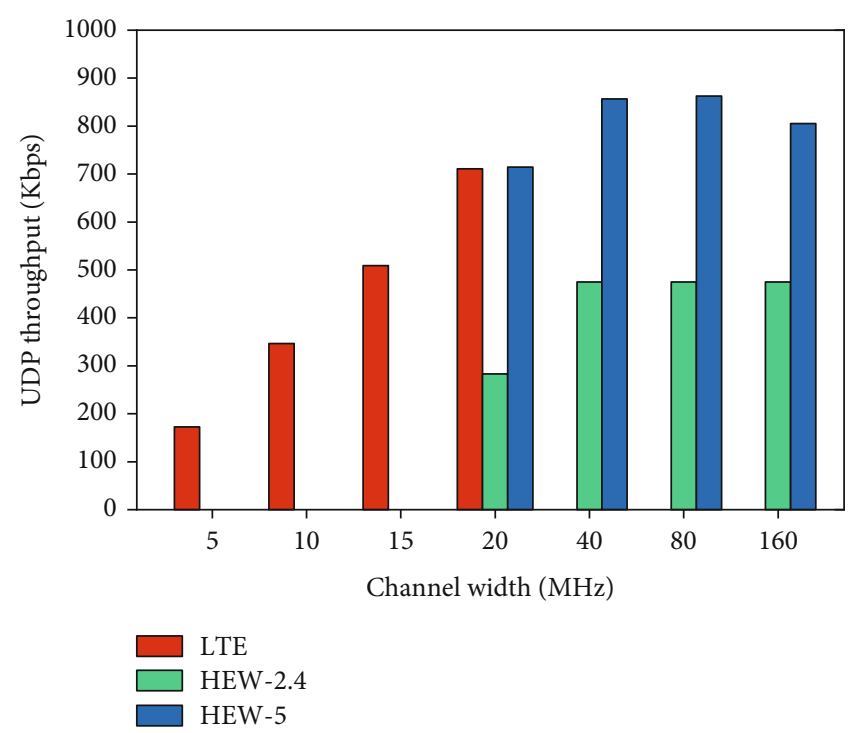

(a)

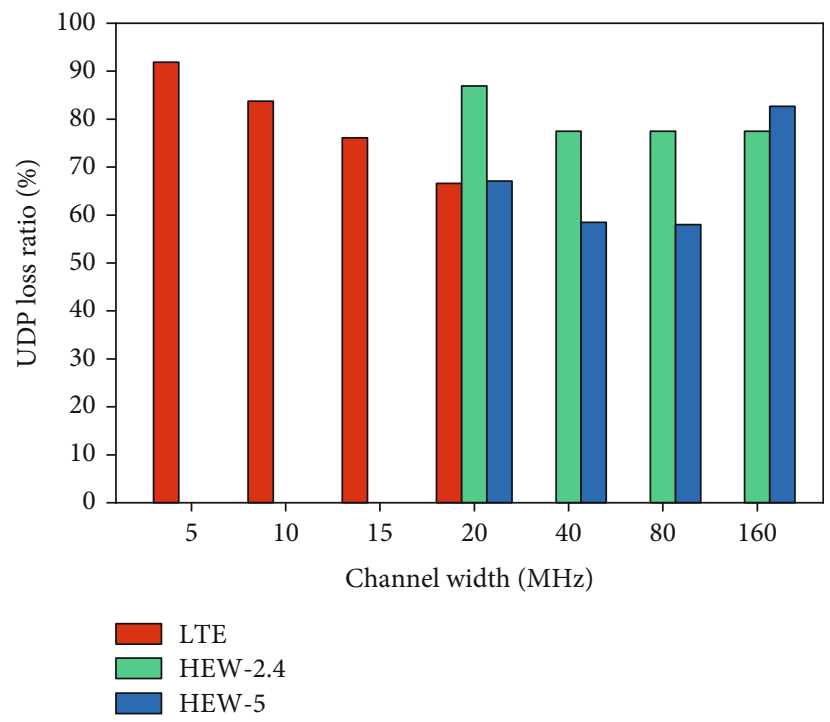

(c)

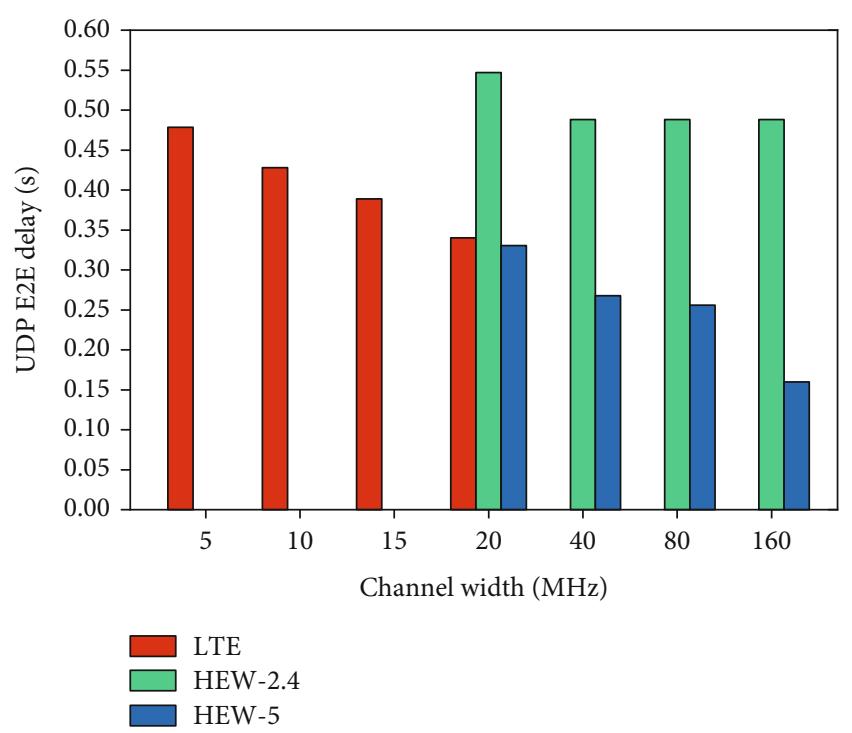

(b)

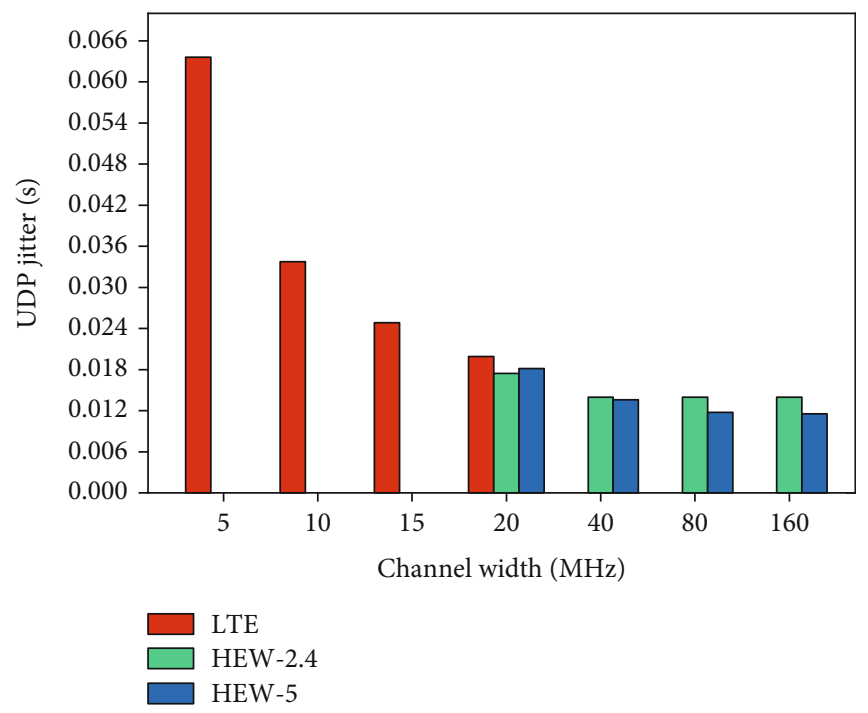

(d)

FIGURE 4: Operating channel width management: UDP (a) throughput, (b) delay, (c) loss ratio, and (d) jitter.

transmission time interval (TTI). Therefore, the number of bits in $1 \mathrm{~ms}$ transport blocks will determine the peak throughput in the physical layer $\left(\mathrm{PT}_{\mathrm{PL}}\right)$ which is calculated as follows:

$$
\mathrm{PT}_{\mathrm{PL}}=\mathrm{TBS} \times N_{\mathrm{SSF}} \times N_{\mathrm{SS}}
$$

where TBS is the transport block size, $N_{\mathrm{SSF}}$ is the number of scheduled subframes, and $N_{\mathrm{SS}}$ is the number of spatial streams in the antenna. As mentioned earlier, TBS is determined by CW, RB, and MCS according to [41]. Accordingly, the TBS values for $5,10,15$, and $20 \mathrm{MHz}$ channels are 18336 , 36696, 55056, and 75376, respectively. Moreover, since in the above formula, the TBS is per $1 \mathrm{~ms}$, and we also have one sub- frame per each TTI $(1 \mathrm{~ms})$; the simplified form of the equation in bps will be as follows:

$$
\mathrm{PT}_{\mathrm{PL}}(\mathrm{bps})=\mathrm{TBS} \times N_{\mathrm{SS}} .
$$

\section{Results and Discussion}

The results from the implementation of high dense LTE, HEW-2.4, or HEW-5 networks in the model are presented in this section. The results assist in the selection of the proper optimal values of different deployment options to enhance the overall network capacity in high-density areas.

4.1. Operating Channel Management. Due to the existence of different channel widths, it is essential to identify their 


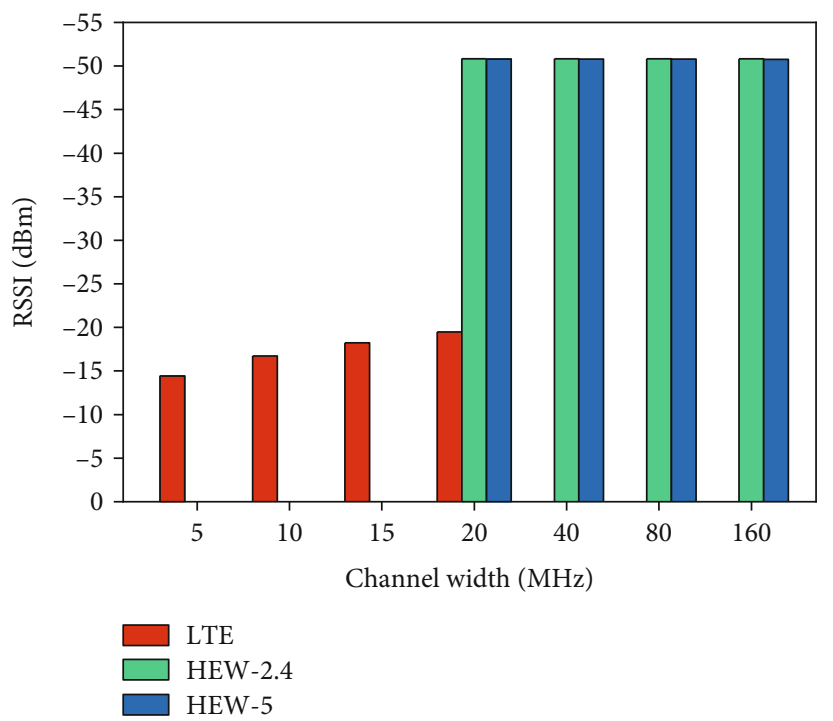

(a)

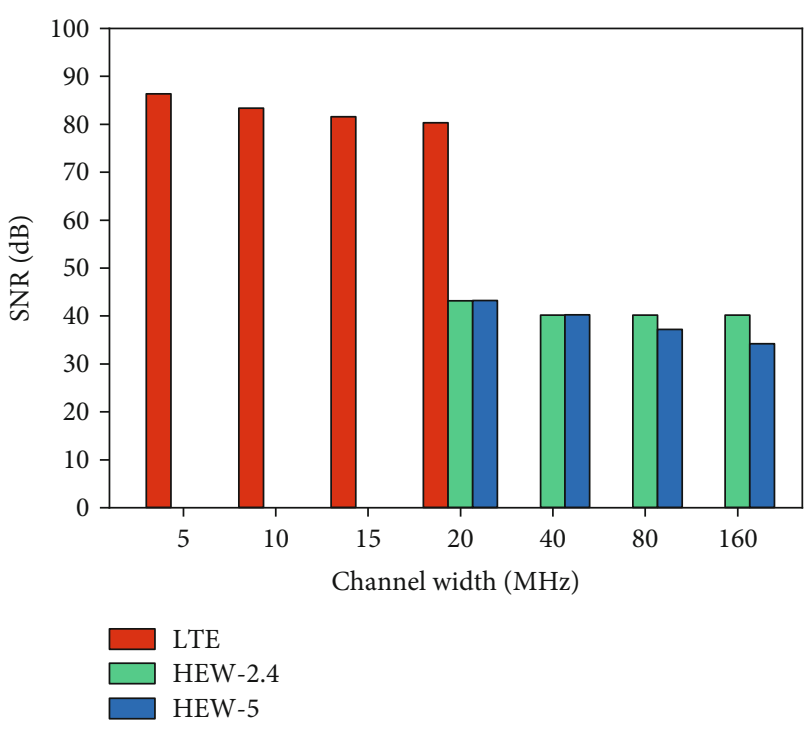

(b)

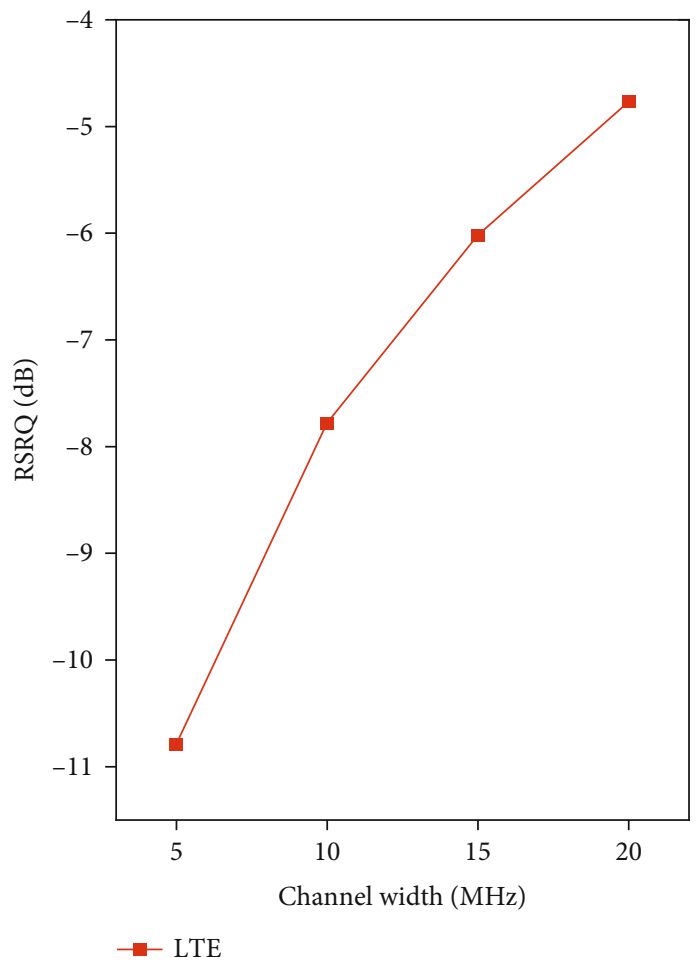

(c)

FIgURE 5: Operating channel width management: (a) RSSI, (b) SNR, and (c) RSRQ.

influence on the performance of LTE and HEW networks and further determine the most efficient operational channel to enhance the capacity of these networks in dense areas. The results regarding the performance of error-sensitive applications are provided in Figure 3.

The results indicate a better performance of HEW over LTE in high-density conditions. The LTE throughput, regardless of the width of the channel, provides the least values compared to HEW-2.4 and HEW-5 networks.
Although by increasing the channel width, the LTE throughput also increases, and it is not considerable. Accordingly, the delay and jitter analysis also show a better performance for HEW-2.4 and HEW-5 compared to LTE so that the wider LTE channels are able to decrease delay, loss ratio, and jitter. Among the four available channel widths, the widest channel $(20 \mathrm{MHz})$ is able to provide the highest performance for the LTE users. Further analysis of the HEW performance shows interesting findings. Regardless of choosing a wide or narrow 


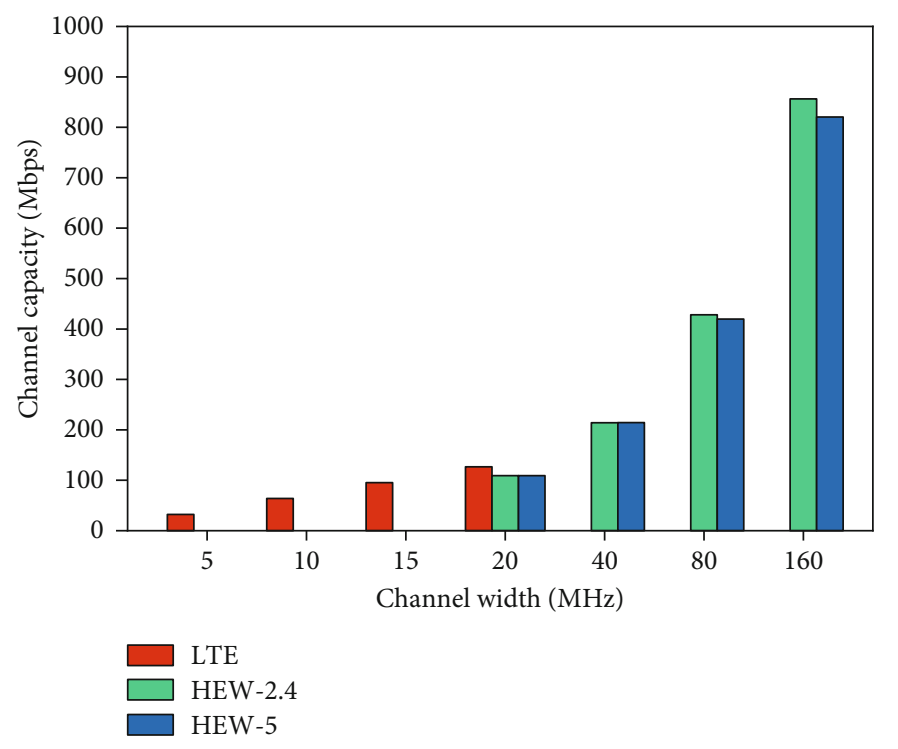

FIGURE 6: Operating channel width management: channel capacity.

channel, HEW-5 outperforms HEW-2.4 in dense areas. With regard to the HEW-2.4 network, among the four available channel widths, increasing channel width from $20 \mathrm{MHz}$ to $40 \mathrm{MHz}$ improves the performance which remains the same for $80 \mathrm{MHz}$ and $160 \mathrm{MHz}$ with no significant changes. In contrast, in the HEW-5 network, the channel width directly affects the overall performance of the end users. In terms of throughput, the throughput increases in line with increasing the channel width with $160 \mathrm{MHz}$ channel as the exception. While the highest capacity for the users connected to the HEW-5 network is achieved by the $80 \mathrm{MHz}$ channel, the least throughput is achieved by the widest channel $(160 \mathrm{MHz})$ due to the lowest number of nonoverlapping channels. The reason is that the channel width controls the speed of data transfer. The wider channels, on one hand, provide higher data rates and consequently, optimize the network performance, but on the other hand, they are subject to more signal interference. Since we implement the network in high-density conditions with 100 active users transmitting simultaneously, this leads to a major interference problem caused by the overlapping channels. Consequently, the $160 \mathrm{MHz}$ with the highest number of overlapped channels results in lower performance in higher frequency $(5 \mathrm{GHz})$. The experimental testbed results in [42] also demonstrate how the $160 \mathrm{MHz}$ channel acts differently. Therefore, for the high dense deployment of $\mathrm{HEW}$ in the $5 \mathrm{GHz}$ frequency band, the $80 \mathrm{MHz}$ channel width for error-sensitive data provides the best performance for the end users. Further work needs to be done to determine whether similar findings are achieved for time-sensitive traffic. Thus, the corresponding scenarios are performed for LTE, HEW-5, and HEW-2.4 networks, and the performance of time-sensitive applications is provided in Figure 4.

As compared to the error-sensitive results, it can be inferred from the results that time-sensitive communications over LTE and HEW networks improve the throughput but degrade the performance of the users in terms of delay, loss ratio, and jitter. This is related to the lower overheads of UDP compared to TCP, which leads to an increasing number of transmitted data. As a result, since more data means more buffering, the higher delay is the unavoidable consequence. Furthermore, by comparing the HEW and LTE, we observe that unlike error-sensitive communication in which the performance of LTE is significantly lower than HEW-5, in timesensitive communication, their performance is very close using the $20 \mathrm{MHz}$ channel width. However, as the width of the channel increases, the HEW-5 performance improves to a higher level than LTE. Thus, it is concluded that for both error-sensitive and time-sensitive traffic exchanged in the LTE network, the wider the channel, the better the performance in high dense areas. With regard to the HEW network, the same conclusion regarding the $160 \mathrm{MHz}$ channel exception is provided. The reason again is the least number of nonoverlapping channels in the $160 \mathrm{MHz}$ width which results in higher interferences under high-density conditions. Since all three networks are under high-density conditions where 100 are actively connected to the same link, and since all users are in close distance from each other, the interference level is very high. In this case, using $160 \mathrm{MHz}$ channels with the lowest number of nonoverlapping channels will make the interference situation even worse resulting in performance degradation.

In order to further validate the findings, a deeper understanding of the high dense requirements in terms of other important considerations including HBLQ parameters is essential. Thus, the corresponding scenarios are run with regard to SNR, RSSI, and RSRQ, and the results are provided in Figure 5.

The RSSI results show that LTE users receive stronger signals compared to HEW users. Moreover, based on the results, the channel width does not affect the strength of the received signals by the HEW-5 and HEW-2.4 users as all 


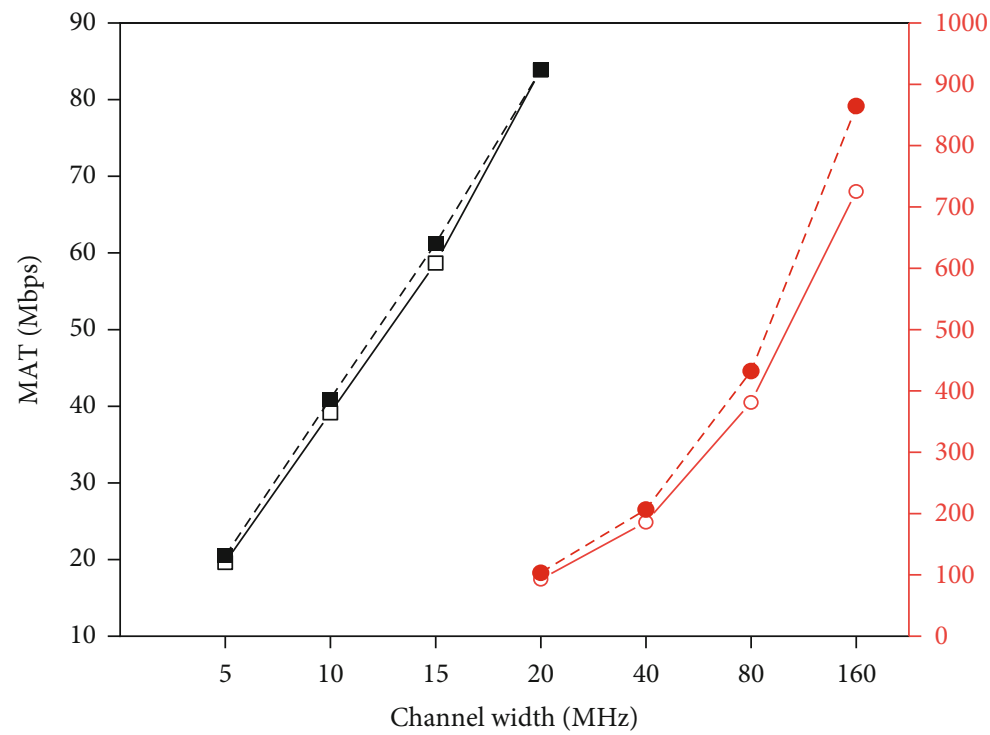

$$
\begin{aligned}
& \text { - - LTE- simulation } \\
& \text { - - LTE- analytical } \\
& \text { - - HEW- simulation } \\
& \text { - - HEW- analytical }
\end{aligned}
$$

FIgURE 7: Analytical and simulation MAT comparison.

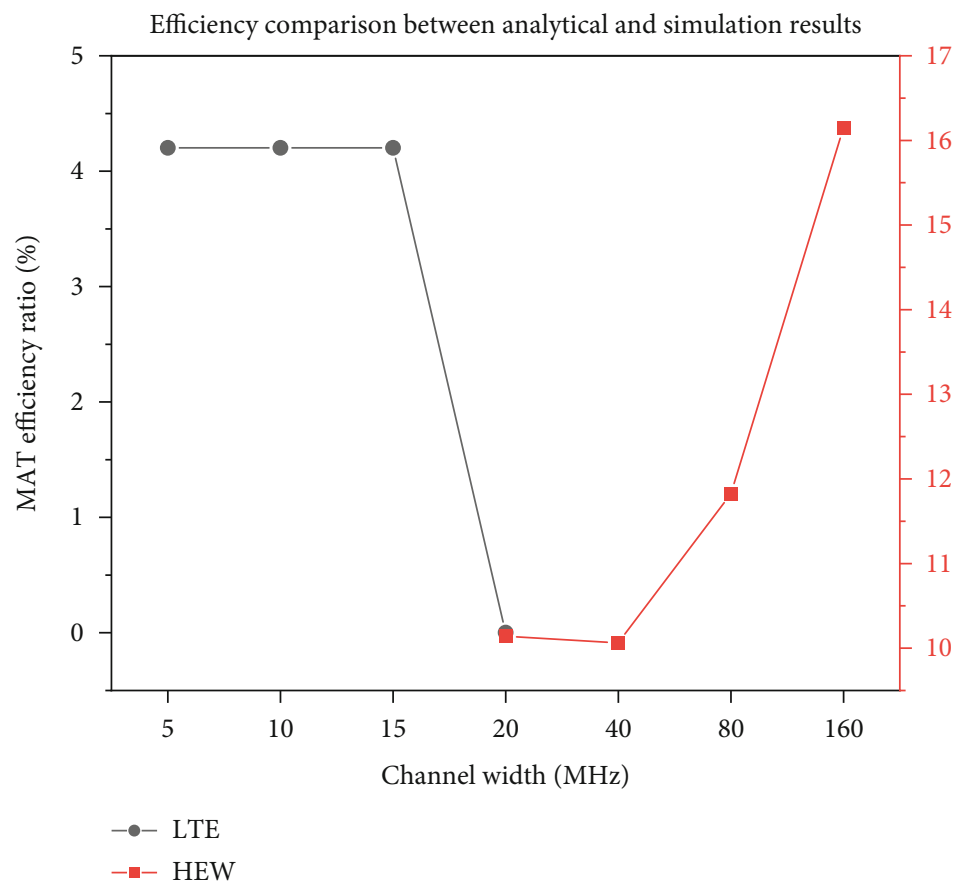

FIgURE 8: Efficiency ratio of analytical and simulation results.

the four available channel widths provide a similar level of RSSI. In contrast, the channel width has a direct influence on the performance of LTE users so that utilizing narrower channels improves the signal in terms of a higher RSSI. Additionally, from the RSRQ results, it is verified that the wider channels are better when the quality of the received signals is the main consideration in LTE dense networks. With regards to SNR results when the noise comes into consideration, different findings are obtained. The SNR results show that while using wider channels leads to SNR reduction, the difference is not remarkable. Because SNR indicates the quality and reliability of the link between the users and the 


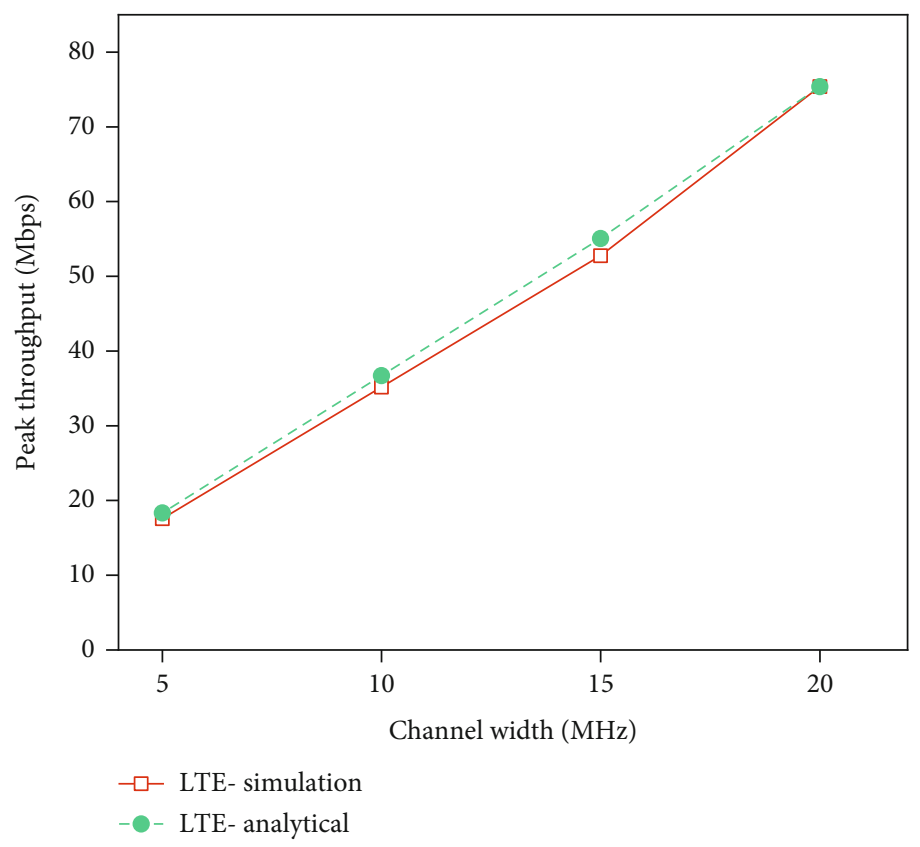

FIgURE 9: Analytical and simulation $\mathrm{PT}_{\mathrm{PL}}$ comparison.

network and because it assists in predicting error occurrence in the networks, to have a better SNR in dense LTE and HEW networks, using narrower channels is more efficient. In this context, it is worthwhile to measure and compare the channel capacity of the LTE, HEW-5, and HEW-2.4 by varying the channel width. The channel capacity results are presented in Figure 6.

The above results indicate the significant impact of the channel width on the channel capacity in LTE and both HEW networks under dense conditions. In the LTE network, as the channel width increases, the channel capacity increase so that the highest amount of channel capacity belongs to the $20 \mathrm{Mhz}$ channels in LTE. Concerning the HEW networks, the HEW-2.4 and HEW-5 achieve the same channel capacity for narrow channels including $20 \mathrm{MHz}$ and $40 \mathrm{MHz}$. However, going toward the wider channels results in different performances for HEW-2.4 and HEW-5 to such an extent that channel capacity is slightly higher for the HEW-2.4 network. The reason is that since in this class of scenario, a single antenna is used in transmitting and receiving sides; according to equation (3), the channel capacity will be only a function of the channel width. Therefore, any increase in the width of the channel directly increases the channel capacity. To further validate the findings, we calculate the analytical maximum achievable throughput $\left(\mathrm{MAT}_{\mathrm{A}}\right)$ values for the LTE and HEW networks and compare them to the simulation MAT $\left(\mathrm{MAT}_{\mathrm{S}}\right)$. The comparison results are presented in Figure 7.

On the basis of the results, both analytical and simulation results confirm a good agreement proven by achieving a better performance using the wider channels. In the LTE network, the measured simulation $\mathrm{MAT}_{\mathrm{S}}$ and analytical $\mathrm{MAT}_{\mathrm{A}}$ are significantly close so that they approximately imply identical values that validate the model as well as the findings.
With regard to the HEW networks, we similarly observe very close values of simulation $\mathrm{MAT}_{\mathrm{S}}$ and analytical $\mathrm{MAT}_{\mathrm{A}}$ while the difference is higher when it comes to the $160 \mathrm{MHz}$ channel width. In this case, the difference between $\mathrm{MAT}_{\mathrm{A}}$ and $\mathrm{MAT}_{\mathrm{S}}$ is higher than those measured for the other channels due to lower nonoverlapping channels considered in the simulation as we explained earlier. Further on this, we obtain the efficiency ratio between the analytical and simulation results $\left(\mathrm{ER}_{\mathrm{AS}}\right)$ demonstrated in Figure 8.

A lower efficiency ratio is an indication of the higher accuracy of the simulation results because of the difference reduction with the analytical results. In this regard, if the efficiency ratio gets closer toward $100 \%$, it means the difference between the simulation and analytical results increases which is not desirable. By looking at the $\mathrm{ER}_{\mathrm{AS}}$ results in the LTE network, we observe $0 \%$ for the largest channel width $(20 \mathrm{MHz})$ which means that the simulation results exactly match the analytical results. However, for narrower channel widths (5, 10 , and $15 \mathrm{MHz}$ ), the $\mathrm{ER}_{\mathrm{AS}}$ value increases to about $4.2 \%$. In this case, despite being higher than $\mathrm{ER}_{\mathrm{AS}}$ for $20 \mathrm{MHz}$, the value is still very low indicating a high precision level of the simulation measurements as they are matched closely with the analytical results (95.8\% match). With reference to the HEW results, it is found that the highest level of accuracy $\left(\mathrm{ER}_{\mathrm{AS}}=0 \%\right)$ is achieved for the narrower channels (20 and $40 \mathrm{MHz}$ ). Then, by increasing the channel width, the $\mathrm{ER}_{\mathrm{AS}}$ increases as well so that the highest difference between the simulation and analytical results belongs to HEW networks that utilize the $160 \mathrm{MHz}$ channel width $\left(\mathrm{ER}_{\mathrm{AS}}=4.3 \%\right)$. These very low ratio values confirm the accuracy of the simulation results. Next, we need to assess the degree to which the measured simulation results are a representation of the analytical results from the perspective of the channel width. Thus, we extend the analysis by comparing the simulation peak 


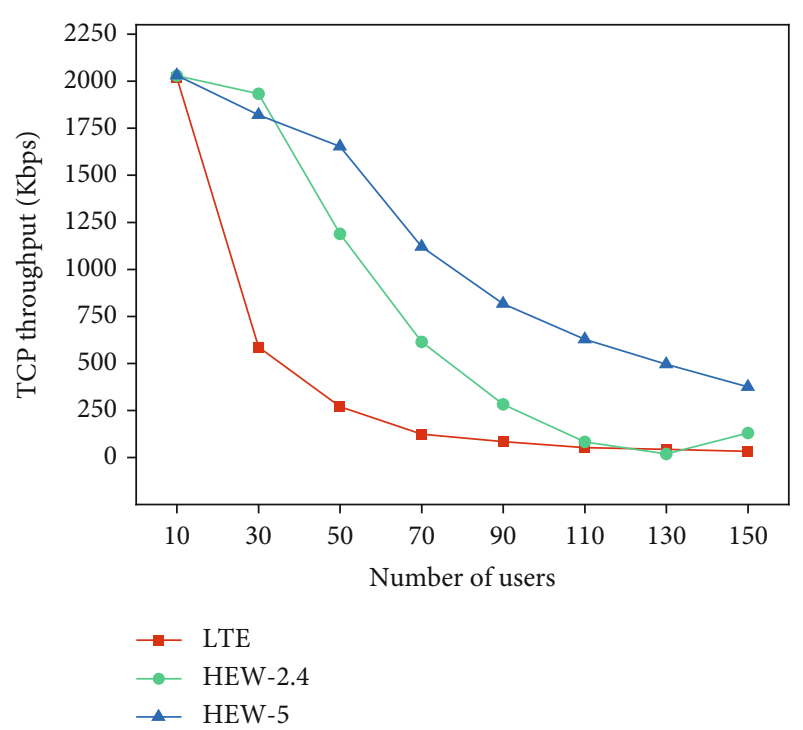

(a)

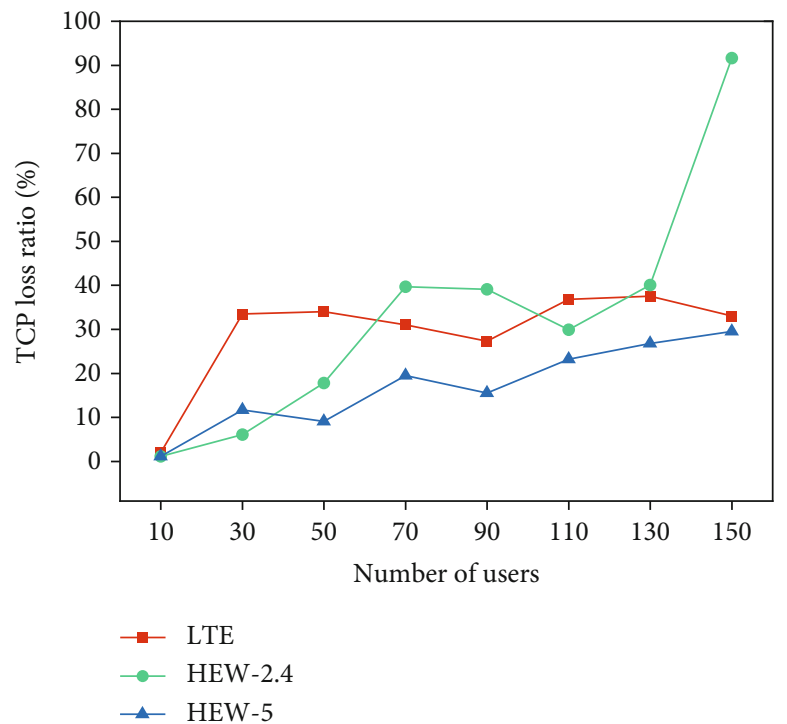

(c)

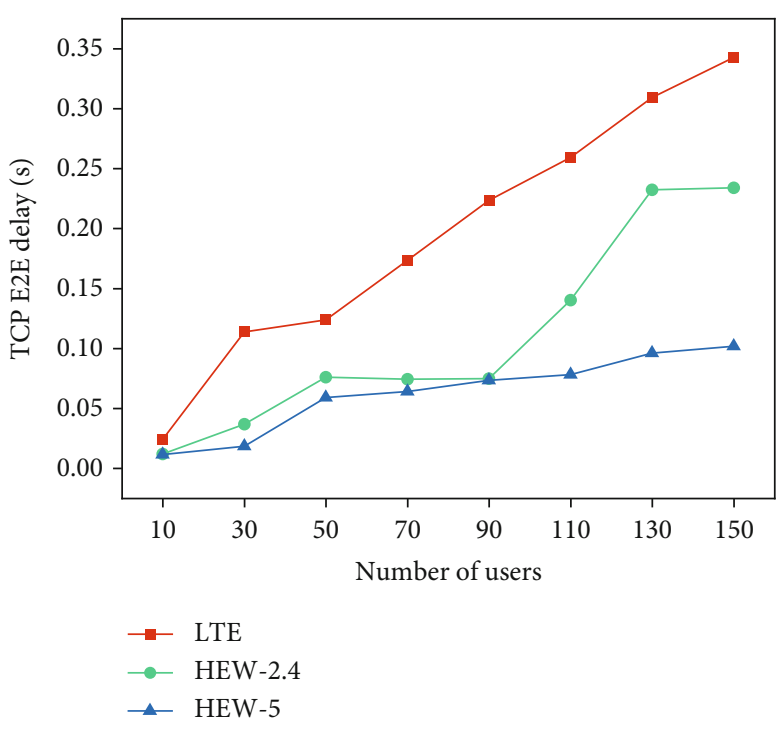

(b)

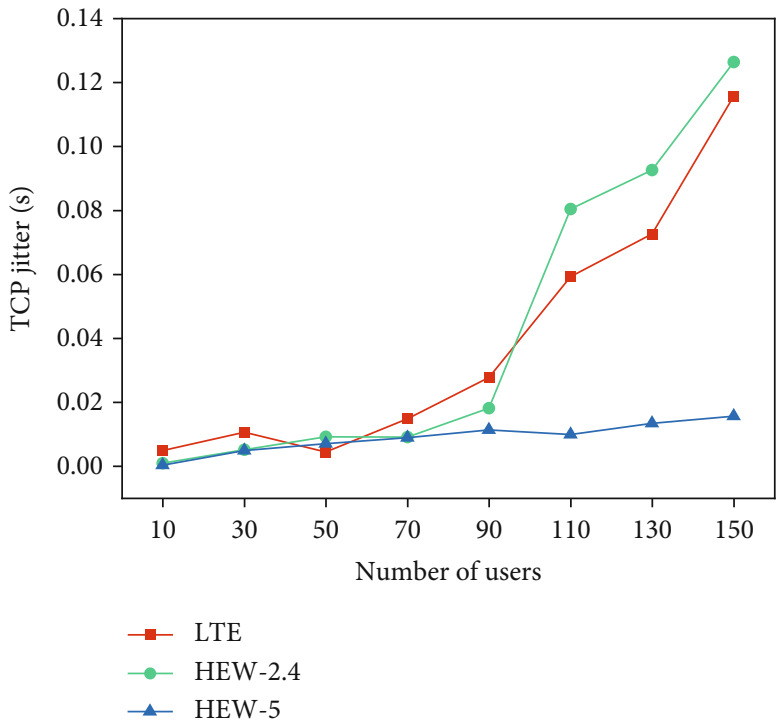

(d)

FIGURE 10: Maximum connection limit: TCP (a), throughput (b), delay (c), loss ratio, and (d) jitter.

throughput with those of the analytical in the physical layer. The comparison results are demonstrated in Figure 9.

The analytical peak throughputs concur very well with the corresponding simulation results to confirm our earlier findings. The number of bits transmitted by transport blocks as the physical layer payload is higher when the $20 \mathrm{MHz}$ channel width is used. This represents the $20 \mathrm{MHz}$ channel width as the most efficient among the other LTE channels to be deployed in high-density areas. Moreover, the very close values of the simulation and analytical peak throughput results further confirm the validity of the findings.

4.2. Maximum Connection Limit. Considering the growing number of connected devices, further analysis is performed here to resolve how LTE and HEW networks can handle the increasing number of simultaneous connections. We vary the number of users joined to the LTE, HEW-2.4, and HEW5 to create small, medium, and high dense areas and measure the corresponding influences on the overall performance of the users. The results are used to identify the optimum number of users before the networks start losing the ability to provide designated services efficiently. The results for errorsensitive applications are provided in Figure 10.

The results show that the performance of the users in all three networks degrades considerably as the number of users increases in such a way that the degradation is more significant for LTE than HEW. When the number of users is below 30, the LTE achieves the same level of performance as HEW2.4 and HEW-5. However, increasing the number of users above 30 drops the LTE performance to about one-fourth 


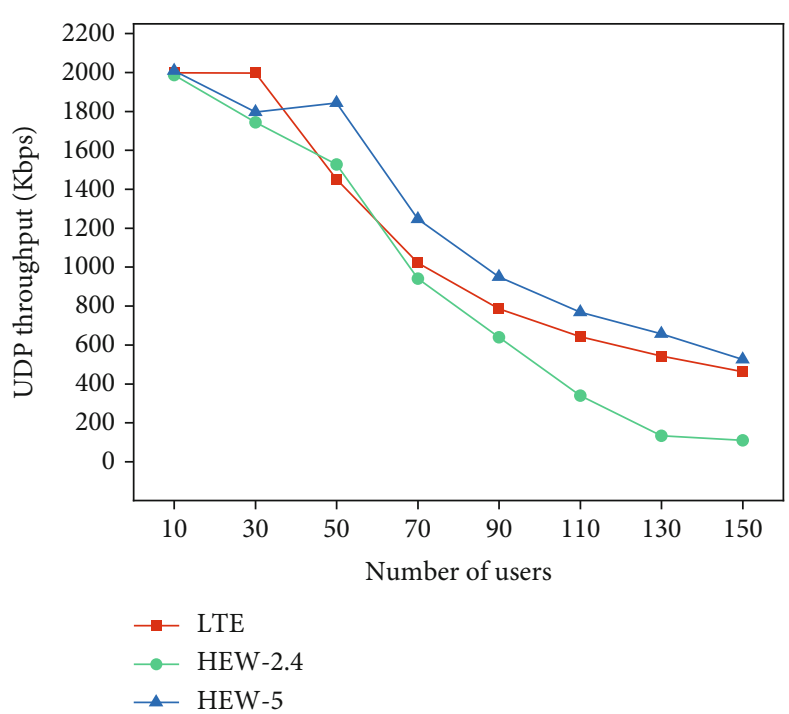

(a)

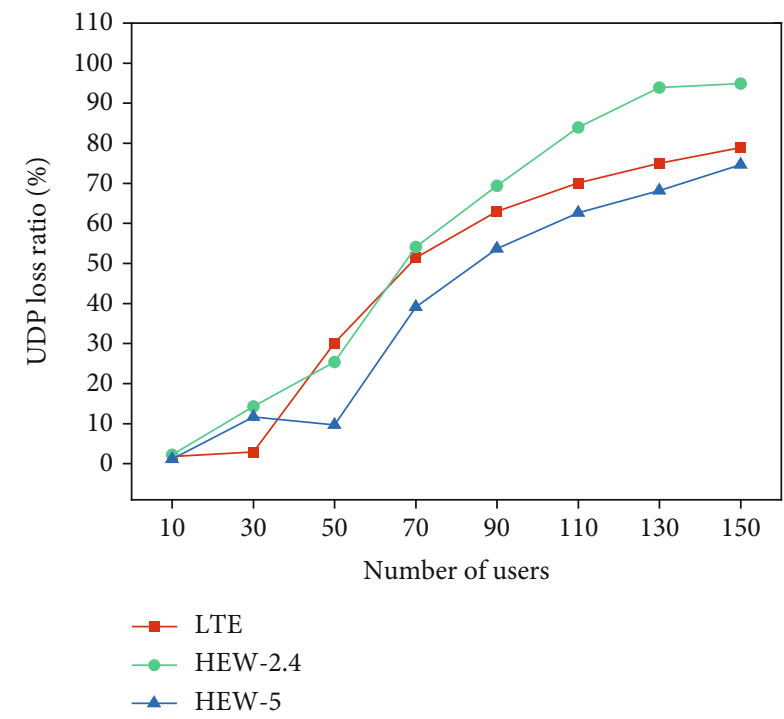

(c)

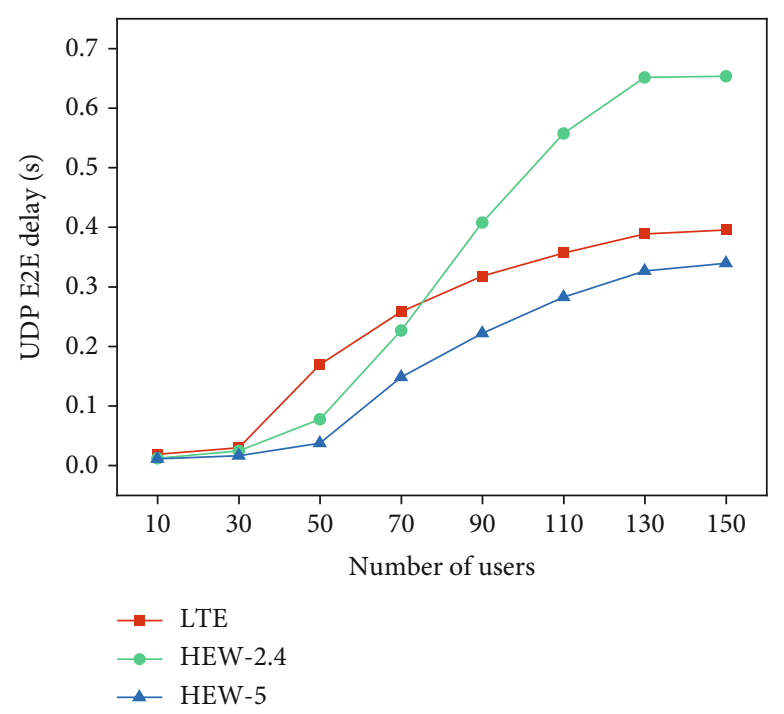

(b)

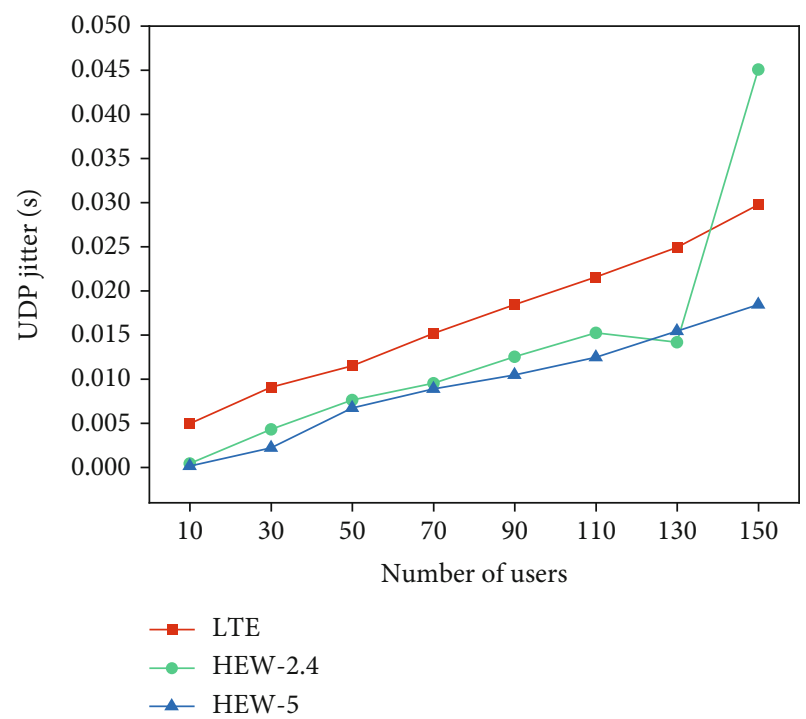

(d)

FIgURE 11: Maximum connection limit: UDP (a) throughput, (b) delay, (c) loss ratio, and (d) jitter.

of what it was before. The performance continues to decline until the number of users reaches 110 and from this point onward, the network is practically unable to provide any services for the error-sensitive applications. The reason is that the LTE network in the model consists of a single macro cell (comparable to the single access point for the HEW network in the model) to which all the users are connected. On the other hand, as mentioned earlier, the resource blocks (RBs) are assigned to users for transmission every TTI ( $1 \mathrm{~ms})$. The number of RBs is finite. Since in this class of scenarios, the channel width is $20 \mathrm{MHz}$, and the total number of RBs is 100. Accordingly, the eNodeB has $100 \mathrm{RBs}$ for the total of $N$ users, and it decides how many RBs are assigned to each user. When there is a lower number of users, more users will have more RBs which provides higher throughput. However, as the number of users increases, there exists fewer users with enough RBs because more active users concurrently share the total cell capacity. Clearly, there can be situations in which some users are not assigned any $\mathrm{RB}$. This will result in throughput degradation. The issue gets even worse with TCP transmissions on the network with all the extra overheads compared to UDP. These results are also confirmed in the MATLAB simulator [43] and experimental testbed [44]. Consequently, based on the results, the connection limit of the LTE network is identified up to 30 to ensure reliable services for the error-sensitive data.

In HEW networks, the results indicate better handling of the high number of simultaneous connections in HEW-5 compared to the HEW-2.4 network. Both networks provide services similarly when the number of connected users is under 50 and then from this point onwards, the difference starts. By growing the number of connections from 70 to 


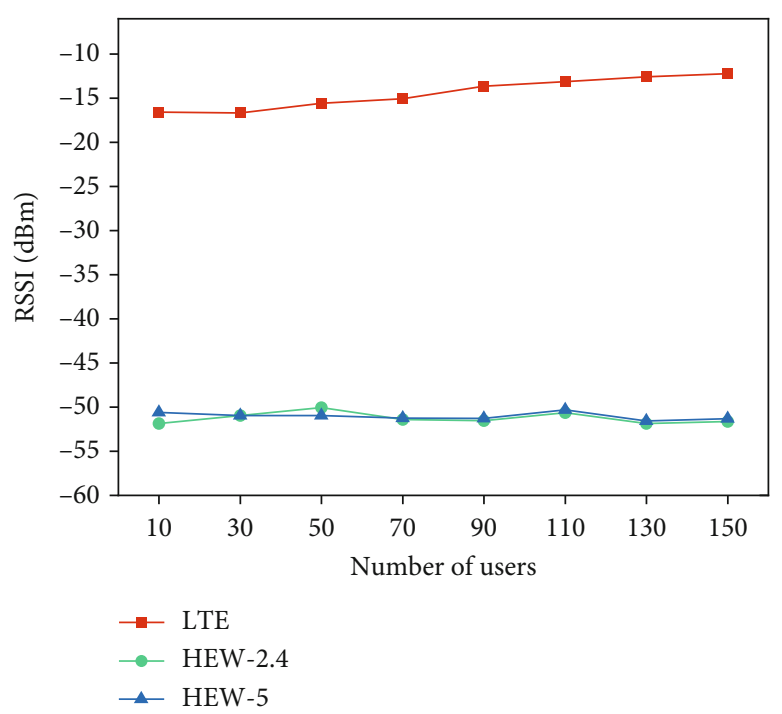

(a)

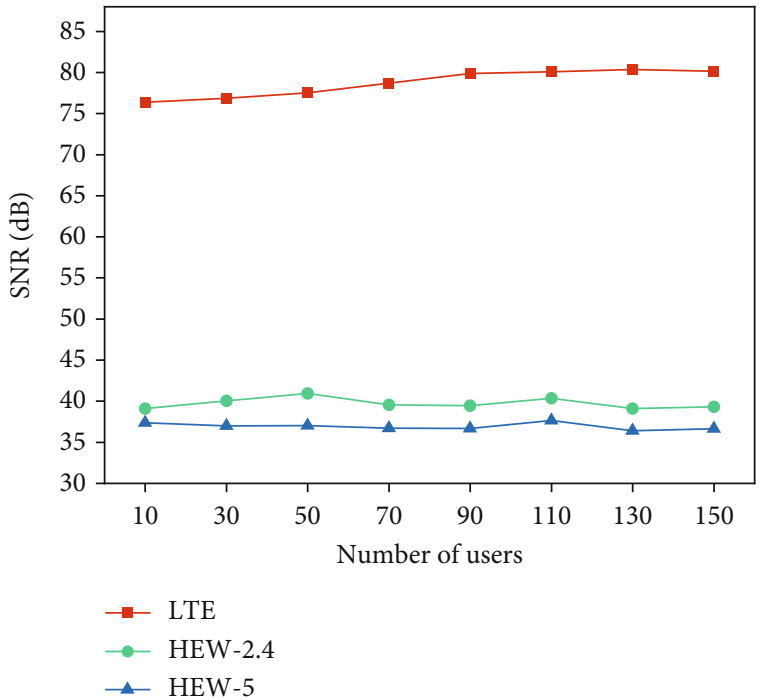

(b)

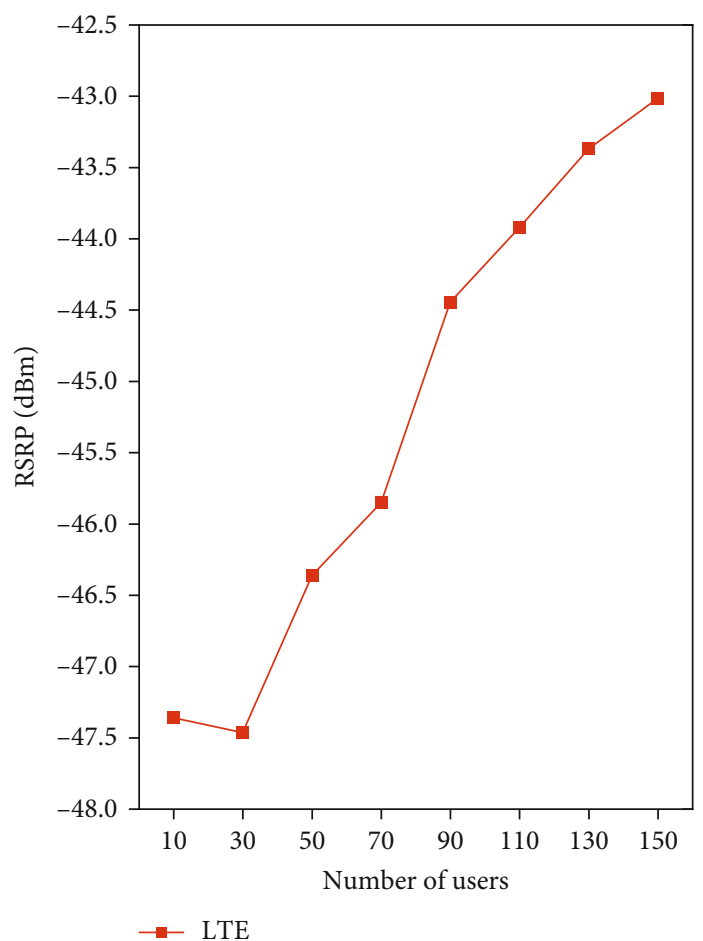

(c)

Figure 12: Maximum connection limit: (a) RSSI (b) SNR (c) RSRP.

150, the performance reduction of HEW-2.4 and HEW-5 starts in such a manner that for 110 simultaneous connections and above, the HEW-2.4 fails to provide its designated services while HEW-5, despite the low performance, still continuously servicing the users even in very high dense conditions with 150 connected users. Therefore, the obtained results provide evidence that the HEW-2.4 and HEW- 5 networks succeed to handle up to 50 simultaneous connections in not high but an acceptable level. These results are completely matched with Cisco documents that recommend around 50 users per access point to ensure an average quality of experience in high-density deployments [2]. We now consider changing the application traffic type to support timesensitive services in LTE, HEW-2.4, and HEW-5 networks, and the results of which are provided in Figure 11.

Analysis of the results shows that unlike the significant difference between high dense LTE and HEW networks in the presence of error-sensitive applications, the difference is not significant when time-sensitive data is exchanged in the networks due to less UDP overheads. All three networks 
handle the high-density connections similarly, and a close performance is achieved for their end users. A closer look at the data indicates how important it is to know the connection limit of each network. As more users are connected to LTE and HEW networks, despite their design purposes to handle high-density conditions, they can achieve it only to some extent. The results identify the optimal number of users as 90 to deliver time-sensitive services to the LTE, HEW-2.4, and HEW-5 end users at an acceptable level of performance. By connecting more users above this limit, users' satisfaction is on the decline in all three networks while the users in the LTE dense network are the least satisfied. We now turn to the analysis of the capacity issue concerning HBLQ parameters to determine whether the differences between LTE, HEW-2.4, and HEW-5 networks are statistically significant. The results are found in Figure 12.

The results indicate that increasing the number of users does not affect the received signal strength. The RSSI levels in LTE, HEW-2.4, and HEW-5 networks remain almost the same with only small changes as the number of users increases. In this context, significant degrading of the signal strength is not observed as a result of the growing number of users, and their corresponding RSSI levels remain in the normal range. Likewise, the SNR results are consistent with the RSSI results. The SNR in LTE, HEW-2.4, and HEW-5 networks does not significantly vary as more users connect to the networks and remain in a normal range. Moreover, there is no statistically significant difference between the SNR levels of the users in HEW-2.4 and HEW-5 networks. In contrast, measuring the signal power received by the users shows that the RSRP level is affected in the LTE network as the number of users increases. Although the difference is not significant, the RSRP level increases as the number of users increases. To determine the starvation management by the HEW and LTE under high dense conditions, the fairness index (FI) is measured, and the results are presented in Figure 13.

As already mentioned, the HEW standard designed particularly for dense networks, and the above FI results confirm this for the $5 \mathrm{GHz}$ frequency band but not in $2.4 \mathrm{GHz}$. Based on FI results measured in the HEW-5, as the network becomes denser with more and more users, it successfully provides the highest level of fairness for sharing the network resources among the users. In this case, even for the highest number of users when $N=150$, the FI remains close to one which represents $100 \%$ fairness. With regard to the LTE network, although the provided fairness is lower than the HEW5 , it is still very high so that in the worst case when 150 users simultaneously communicate in the network, the FI is close to 0.9 representing $90 \%$ fairness for the users. in contrast, the lowest fairness is provided in the $2.4 \mathrm{GHz}$ frequency band in HEW. When the number of users is below 50, the HEW2.4 is able to provide a fairness level equal to the LTE. However, after that, as more users join the network, the contention increases, and inevitably, the users get a less fair share so that for $N=150$, the achieved fairness is only about $10 \%$. This also strongly confirm the Cisco recommendation as the network density of up to 50 users per AP can provide optimal performance. The results are also consistent with

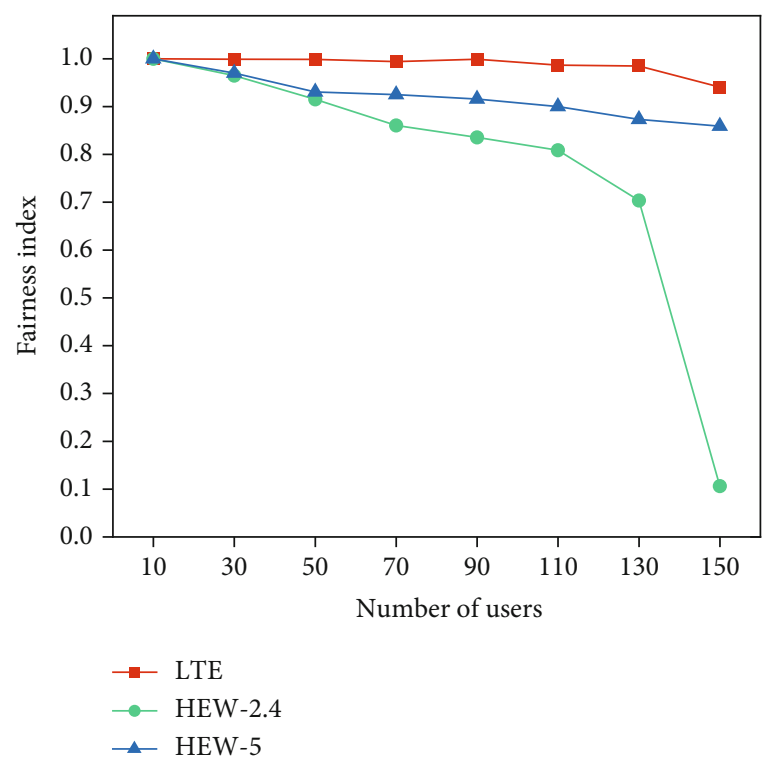

FIgURE 13: Fairness index in the LTE and HEW networks.

the previous findings as better performance of the HEW5 network compared to LTE and HEW2.4 in terms of ensuring a higher level of fairness for its associated users. The HEW5 users experience more fairness than HEW2.4 users due to utilizing a higher frequency which offers faster service delivery for the users.

4.3. Antenna Configuration. The choice of antenna technology and the corresponding configurations related to the transmission modes can affect the performance of the networks. Thus, having special requirements of high-density networks in mind, a decision needs to be made in this regard when deploying mobile networks. This class of scenarios mainly attempts to determine the efficiency of the transmission modes and identify the most suited mode in high dense LTE, HEW-2.4, and HEW-5 networks. The results of exchanging error-sensitive data using different antenna transmission modes are provided in Figure 14.

The findings provide insights for significant improvement accomplished by the LTE in the third mode (OLSM). In this mode, the data is divided into two separate streams that are transmitted simultaneously via two antennas. In contrast, the performance attained by the first mode with one antenna, and the second mode with two antennas is equivalent with no considerable differences. The reason is that, although the LTE second mode includes two antennas to increase data transmission reliability, they are used to transmit two copies of the same data. This is why no increase in the number of transmitted bits is observed. Thus, due to increasing reliability and decreasing error probability, this method is suitable for noisy networks for instance under high-density conditions where many users in a close distance are communicating on the same link. When it comes to HEW-2.4 and HEW-5 networks, despite observing much better performance in HEW- 5 compared to the HEW-2.4 network, the results show that changing the antenna 


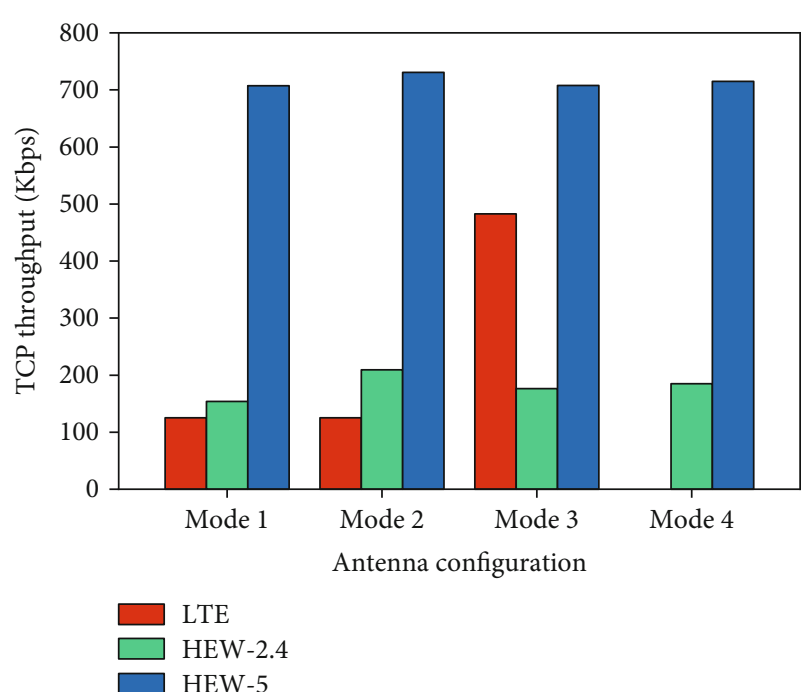

(a)

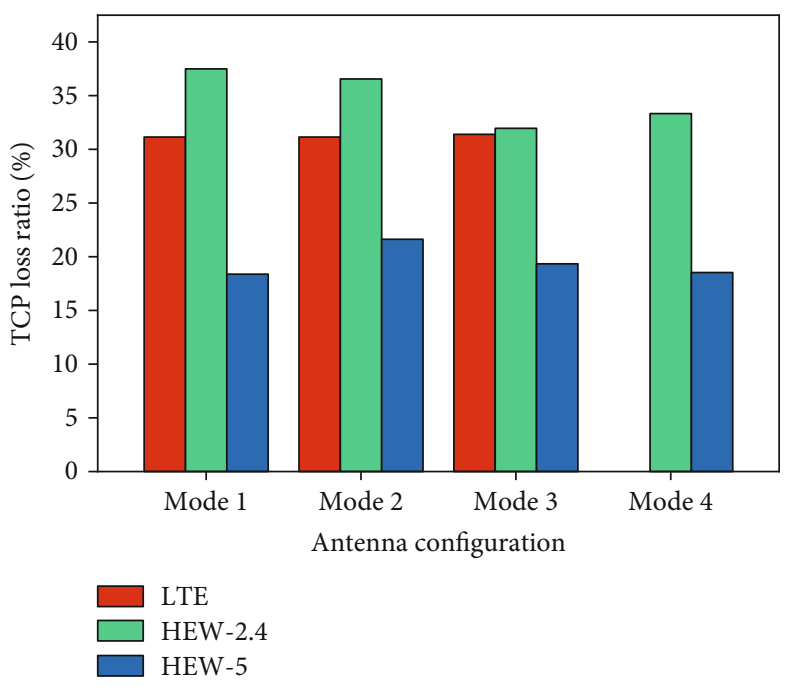

(c)

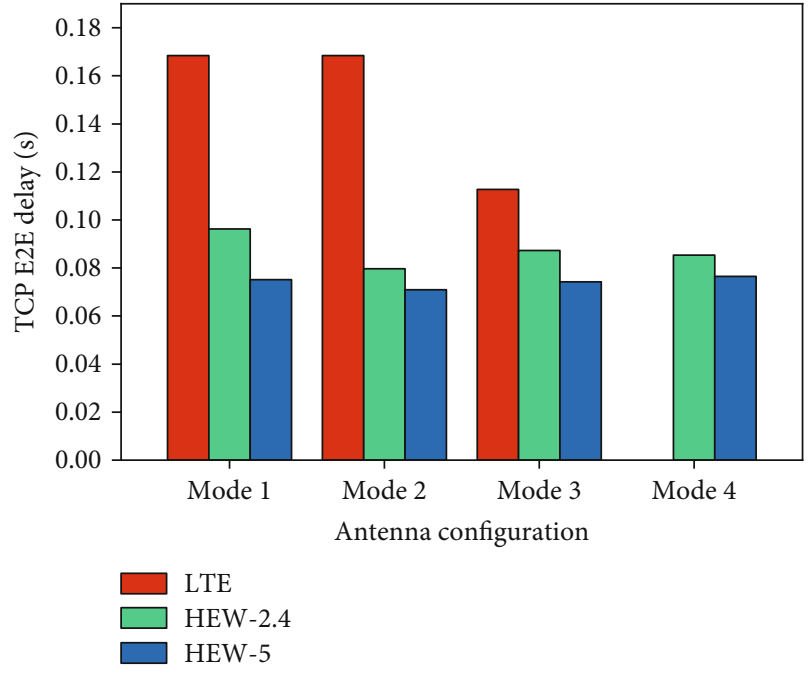

(b)

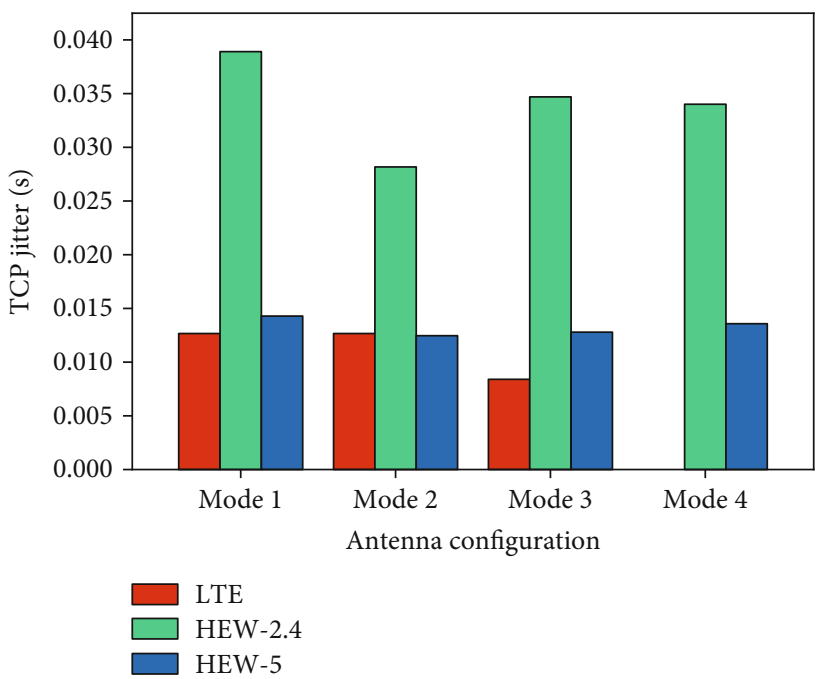

(d)

FIgURE 14: Antenna configuration: TCP (a) throughput, (b) delay, (c) loss ratio, and (d) jitter.

transmission mode does not significantly affect the performance experienced by the users under high-density conditions. Based on the results, the achieved performance by four antennas is similar to when single, two, or three antennas are used, and no considerable changes are detected. This is mainly because the networks are highly congested, and they already reach their maximum bandwidth limit at the saturation level. We further extend the analysis to find out whether the antenna transmission modes affect the performance of LTE, HEW-2.4, and HEW-5 networks in a different way when time-sensitive data is exchanged. The results are provided in Figure 15.

The results prove how the antenna configuration can affect the performance based on the type of applications that exchange data. According to the findings, unlike using errorsensitive applications, when time-sensitive data is exchanged over LTE dense networks, the performance gained by the first and second modes improve highly to a level close to the third antenna mode. Moreover, unlike the error-sensitive results, in which only the third mode can provide higher performance for LTE compared to HEW-2.4, in the presence of time-sensitive applications, all three antenna modes are able to provide better performance in LTE than the HEW-2.4 network. Further, the comparison of HEW-5 and HEW-2.4 implies that similar to error-sensitive applications, HEW-5 outperforms HEW-2.4 in all four available antenna modes. However, unlike before, the performance differences are not significant. Given the results, despite increasing the performance of both HEW-2.4 and HEW-5 networks, due to being highly dense, all four modes accomplish equivalent performance gains. It is important, though, to recognize the relationship between the available antenna transmission modes and their performance in terms of HBLQ parameters. Thus, further analysis is performed in this context beyond SBLQ 


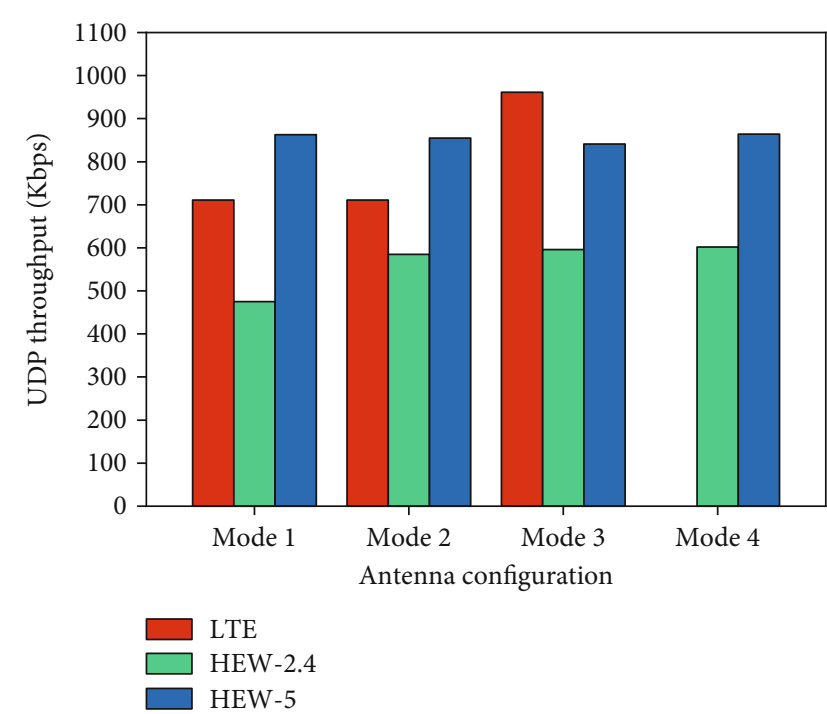

(a)

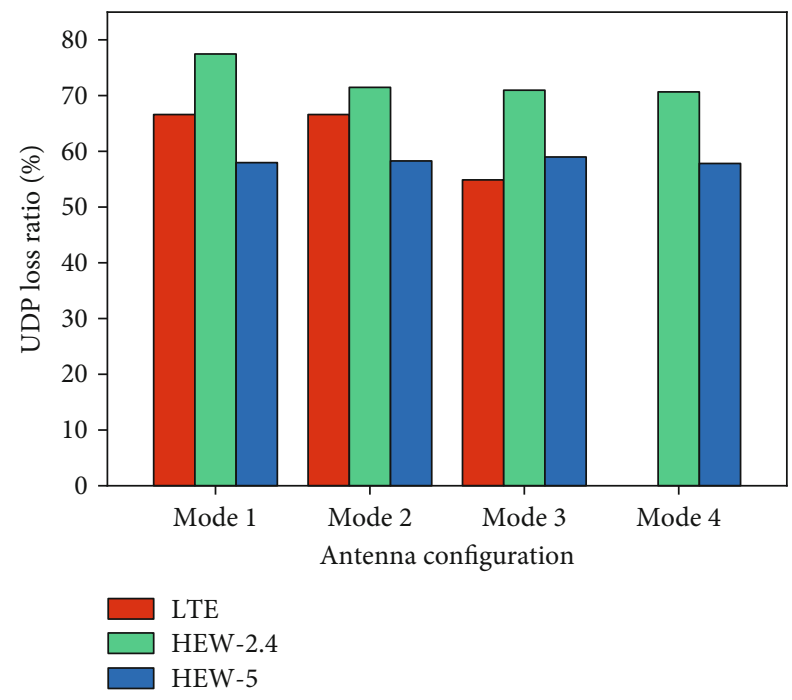

(c)

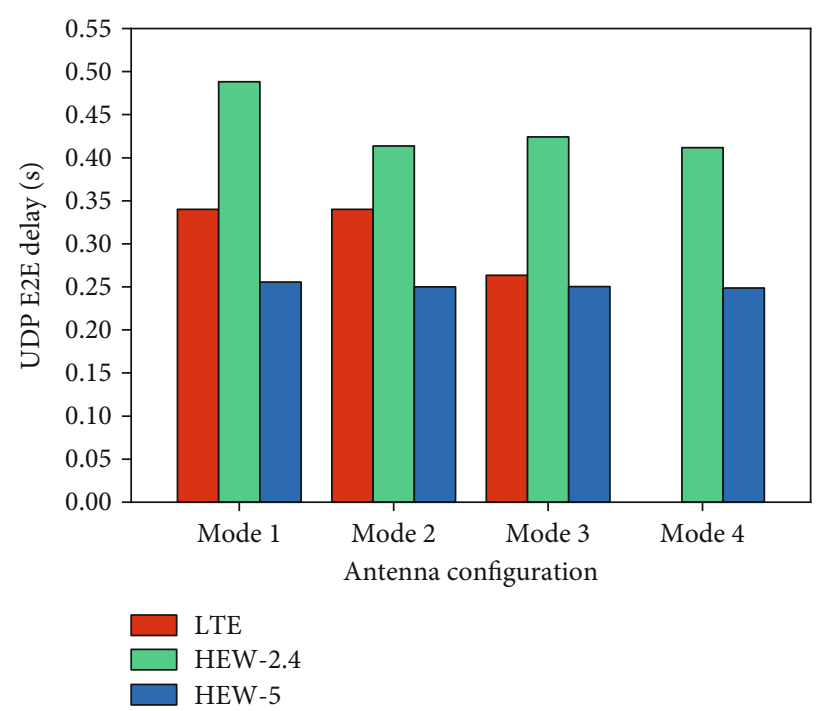

(b)

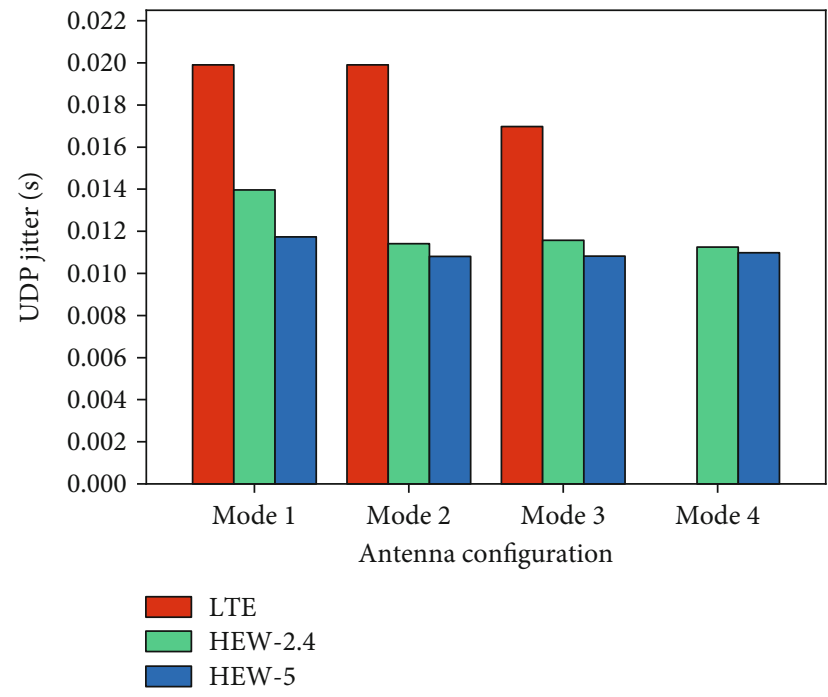

(d)

Figure 15: Antenna configuration: UDP (a) throughput, (b) delay, (c) loss ratio, and (d) jitter.

parameters, and the results of which can be found in Figure 16.

Form the RSSI results, we observe two important findings. First, it can be seen that the strength of the received signal by the users does not depend on the antenna transmission mode in dense areas as all the modes provide similar RSSI levels. Second, no RSSI differences are observed between HEW-2.4 and HEW-5 networks. Furthermore, looking at the SNR results confirms the same findings as the antenna transmission modes in high dense LTE, HEW-2.4, and HEW-5 networks do not significantly affect the SNR values. Even though the SNR provided by HEW-2.4 is higher compared to HEW-5, the difference is negligible. We further take into account the channel capacity to determine the influence of antenna transmission modes on its level, and the results of which are provided in Figure 17.

The results signify the direct impact of antenna configuration modes on the channel capacity of LTE, HEW-2.4, and HEW-5 networks. A substantial improvement of channel capacity is fulfilled when the higher transmission modes are selected to exchange data. Considering the given modes, LTE provides the least channel capacity for its end users in dense areas, whereas both HEW-2.4 and HEW-5 networks provide much higher values. Moreover, the comparison of the HEW-2.4 and HEW-5 channel capacity implies a better performance of the HEW-5 network in terms of higher channel capacity, which is about twice those measured for the HEW-2.4. To further establish a baseline with respect to the accuracy of the simulation results and thereby verify their validity, we provide a comparison of the simulation MAT $\left(\mathrm{MAT}_{\mathrm{S}}\right)$ and analytical MAT $\left(\mathrm{MAT}_{\mathrm{A}}\right)$ in Figure 18.

The above results are consistent with the previous results and confirm three findings. First, the simulation $\mathrm{MAT}_{S}$ results are perfectly matched with the analytical $\mathrm{MAT}_{\mathrm{A}}$ based on providing very similar values. Second, while the first and second transmission modes in LTE provide similar MAT 


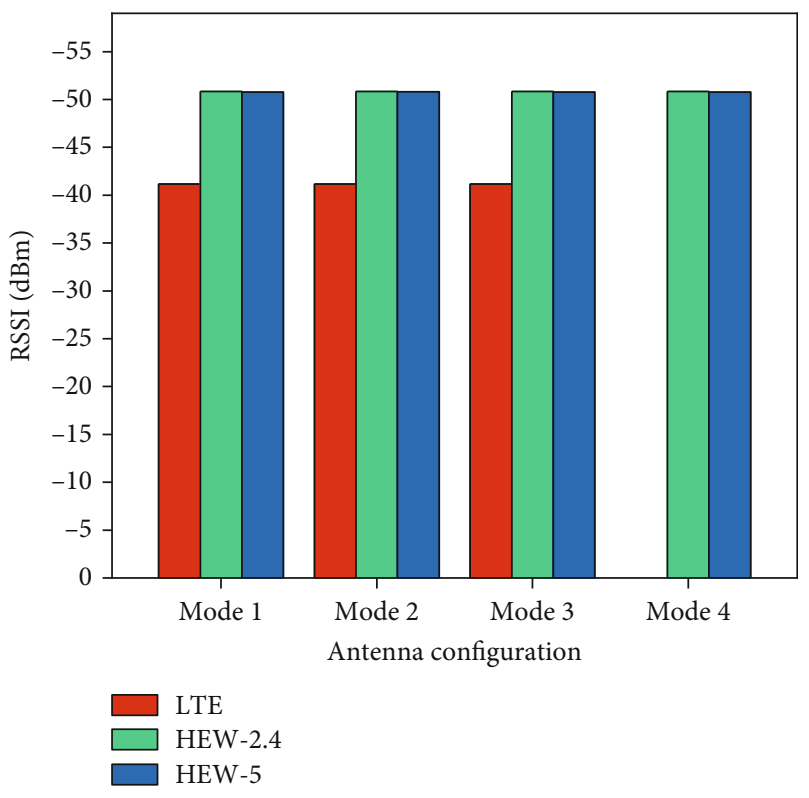

(a)

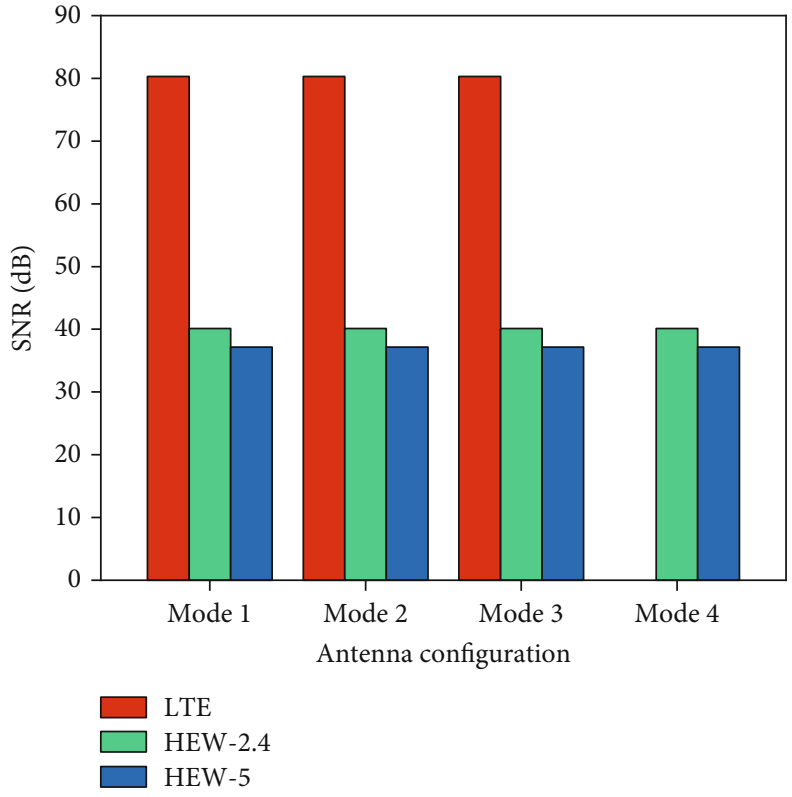

(b)

FIGURE 16: Antenna configuration: (a) RSSI and (b) SNR.

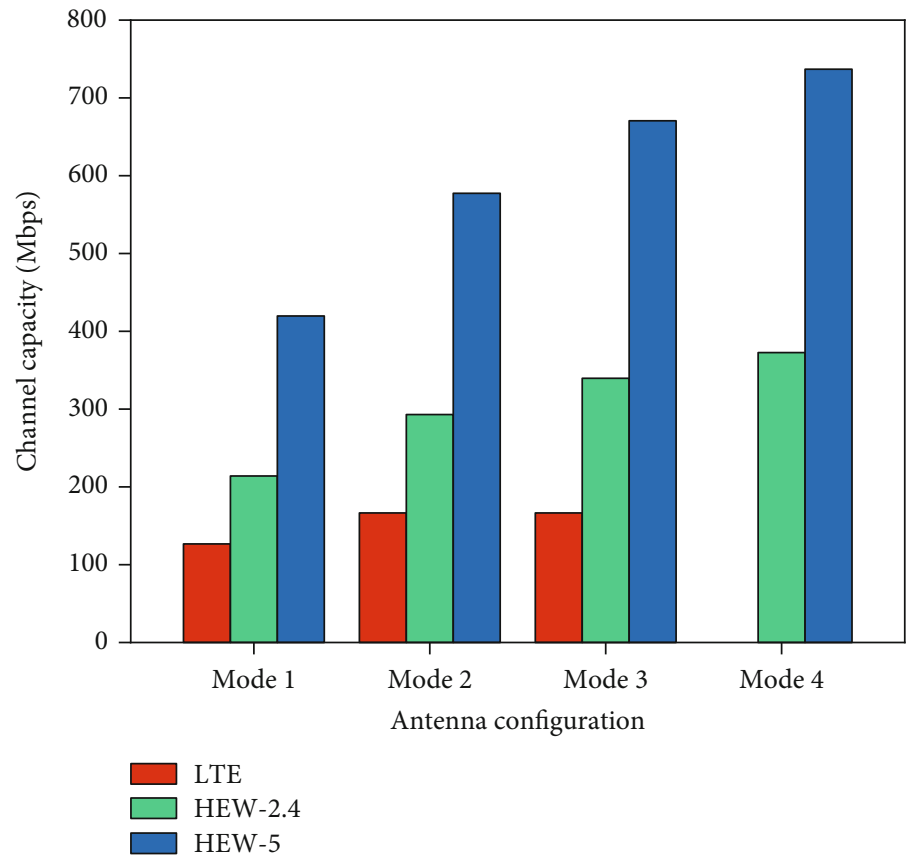

FIgURe 17: Antenna configuration: channel capacity.

values, the third mode provides higher throughput due to the transmission of different data on each antenna. Third, when speed is the main concern in the HEW and LTE networks under high-density conditions, the combination of higher transmission modes with the wider channels can meet the goal. Moreover, to determine if the simulation results meet a satisfactory level of accuracy in terms of consistency with the analytical results, we provide a comparison over the ana- lytical and simulation peak throughputs at the physical layer $\left(\mathrm{PT}_{\mathrm{PL}}\right)$ which is shown in Figure 19.

The side-by-side comparison of the above results, while shows their consistency with the previous findings, also demonstrates a significant agreement between the analytical and simulation results. The obtained results confirm that the maximum peak throughput in the LTE networks under high-density conditions is achieved by the third transmission 


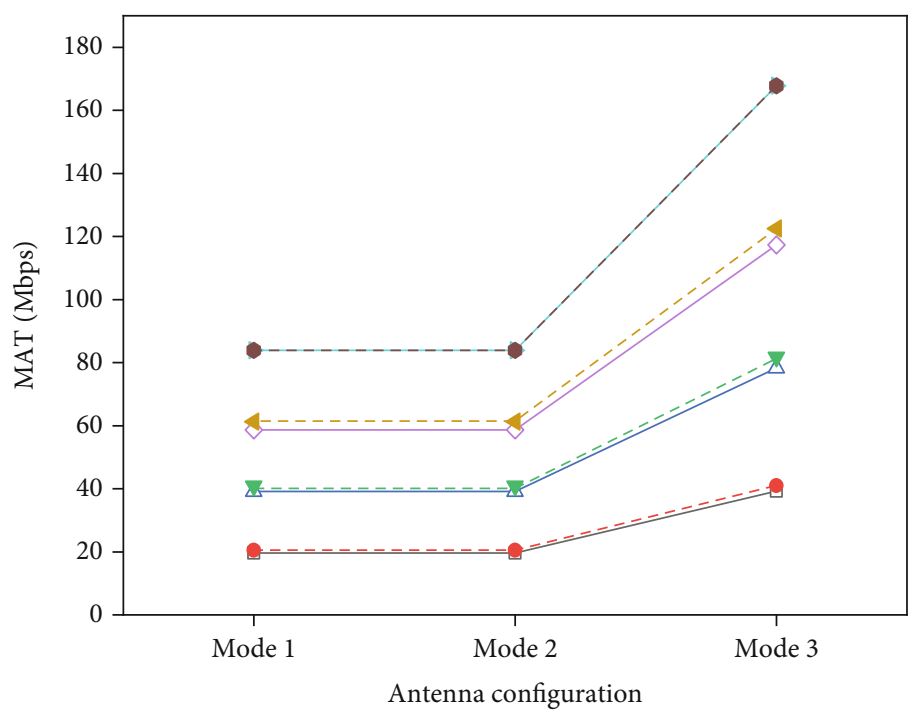

$\neg-$ LTE- simulation $(\mathrm{CW}=5)$

- - LTE- analytical $(\mathrm{CW}=5)$

$\triangle-$ LTE- simulation $(\mathrm{CW}=10)$

$-\nabla-$ LTE- analytical $(\mathrm{CW}=10)$

$\checkmark$ LTE- simulation $(\mathrm{CW}=15)$

$-\triangleleft-$ LTE- analytical $(\mathrm{CW}=15)$

$\rightarrow$ LTE- simulation $(\mathrm{CW}=20)$

- LTE- analytical $(\mathrm{CW}=20)$

(a)

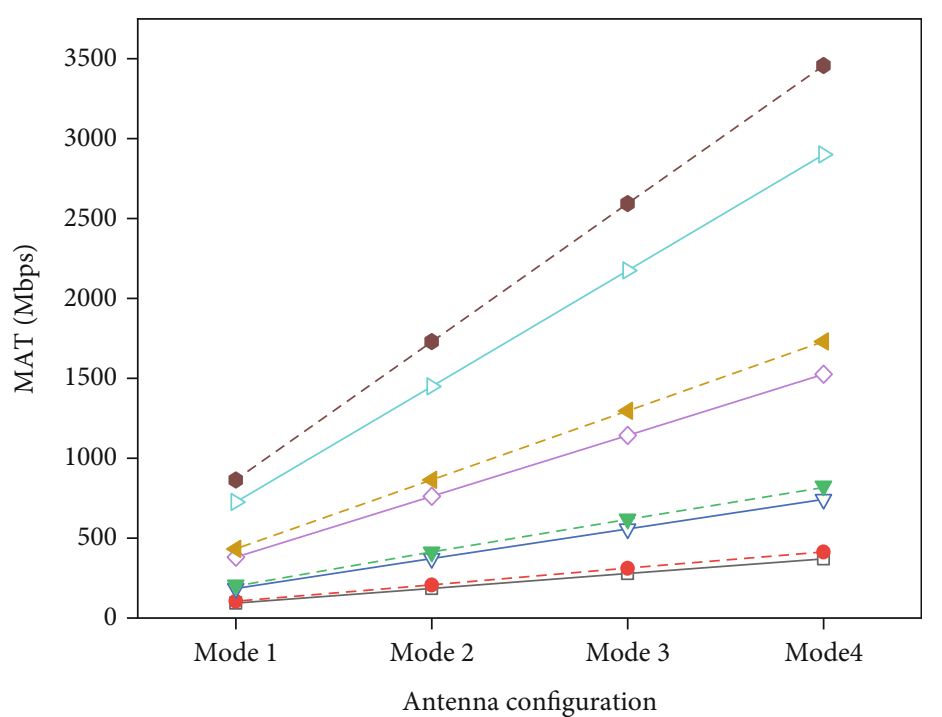

$$
\begin{aligned}
& -\square-\text { HEW- simulation }(\mathrm{CW}=20) \\
& \text { - }- \text { HEW - analytical }(C W=20) \\
& \rightarrow \text { HEW- simulation }(C W=40) \\
& -\nabla-\text { HEW - analytical }(C W=40) \\
& \checkmark \text { HEW- simulation }(\mathrm{CW}=80) \\
& -4 \text { - HEW- analytical }(\mathrm{CW}=80) \\
& \rightarrow \text { HEW- simulation }(\mathrm{CW}=160) \\
& \text { - - HEW- analytical (CW =160) }
\end{aligned}
$$

(b)

Figure 18: Analytical and simulation MAT throughputs: (a) LTE and (b) HEW. 


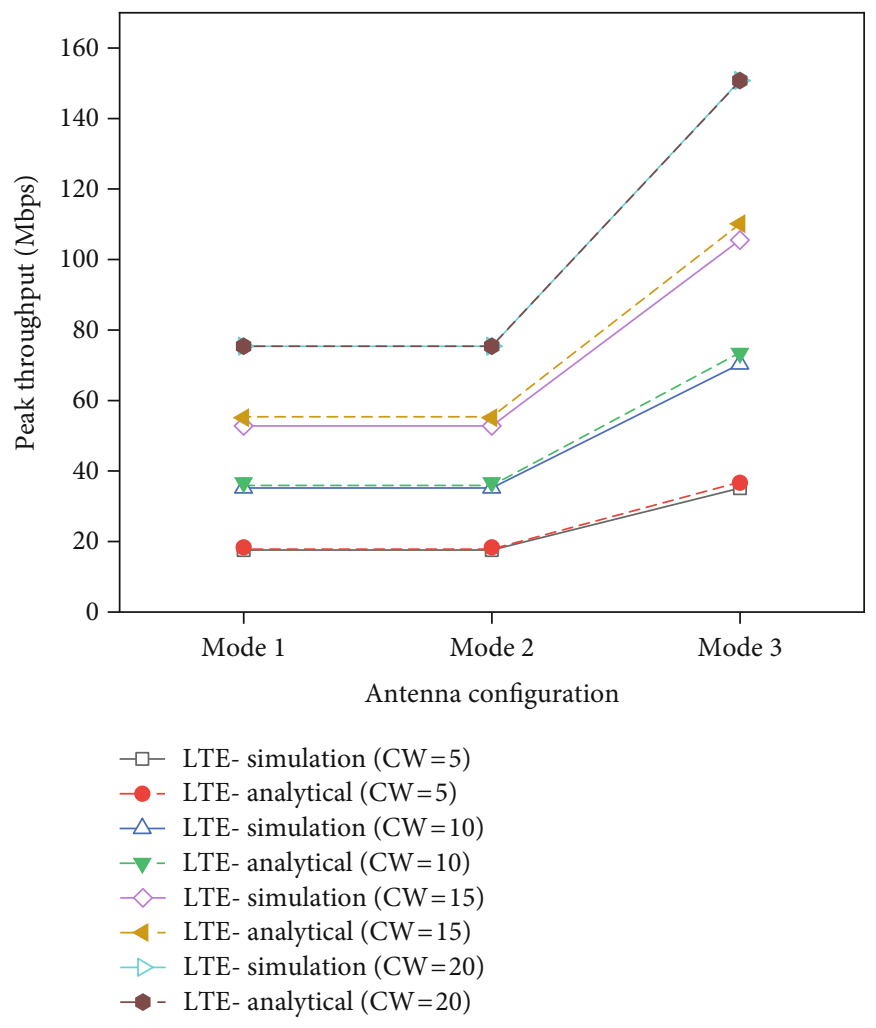

FIGURE 19: Analytical and simulation $\mathrm{PT}_{\mathrm{PL}}$ comparison.

mode in the presence of the largest channel width due to the transmission of two different streams of data on the antennas.

4.4. Internet Protocol Efficiency. Despite the benefits of IPv6, there exist multiple questions regarding its practical functionality in dense areas which leads to ambiguity of IPv6 utilization in the given networks. This demands an in-depth analysis of IPv6 in practice under different operational conditions to be compared with IPv4. Accordingly, we perform a comparative analysis of IPv6-only and IPv4-only to identify the protocol that is more efficient at performance enhancement of LTE, HEW-2.4, and HEW-5 in high-density conditions. The results for error-sensitive applications are provided in Figure 20.

It can be inferred from the results that the slightly better performance of IPv4 compared to IPv6 in the LTE and HEW-5 networks is not considerable, and the two protocols nearly achieve a similar level of performance under highdensity conditions. Thus, the performance gain for errorsensitive applications in the high dense LTE and HEW-5 networks does not rely on the type of network protocol. With respect to the HEW-2.4 network, different results are attained. When IPv4 is utilized for exchanging errorsensitive data, the IPv4 achieves better performance in terms of higher throughput and lower delay and jitter. However, this is in contrast to a better performance of IPv6 in terms of a lower level of lost packets. Therefore, based on the results, when higher speed and lower latency values are the main concerns in high-density HEW networks, IPv4 outperform IPv6. However, when network reliability for optimizing the packet delivery process is important, IPv6 performs better than IPv4 by providing a lower packet loss ratio. The above TCP findings in terms of higher delay and lower loss ratio for IPv6 protocol are also consistent with the experimental testbed results provided in [7, 44-46] under different conditions. Now, to examine if IPv6 can meet the performance requirements of time-sensitive applications, we further extend the analysis and implement both IPv6 and IPv4 in dense LTE, HEW-2.4, and HEW-5 networks. The results are presented in Figure 21.

With regard to LTE and HEW-5 networks, the above results appear consistent with the error-sensitive results and show that there are no significant performance differences between IPv6 and IPv4 for the users in high dense areas. However, from the results of the HEW-2.4 network, we realize that IPv4 outperforms IPv6 with reference to all aspects of performance evaluation parameters. The average delay of IPv4 users in high dense HEW-2.4 is about $0.3 \mathrm{~s}$, whereas the IPv6 users experience an average of $0.5 \mathrm{~s}$. Considering that the time-sensitive applications are highly sensitive to any increased delay, these differences are significant and affect the performance of the applications. Thus, on the basis of the results, while both IPv6 and IPv4 provide similar performance for time-sensitive services in high dense LTE and HEW-5 networks, utilizing IPv4 in HEW-2.4 can improve the performance compared to IPv6. The reason for the lower performance of IPv6 in HEW-2.4 compared to HEW-5 is 


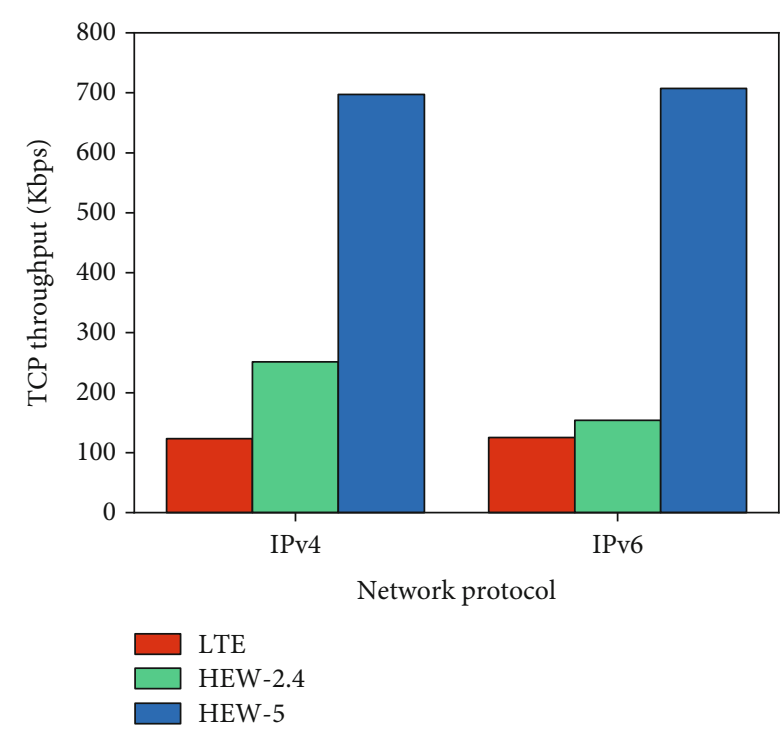

(a)
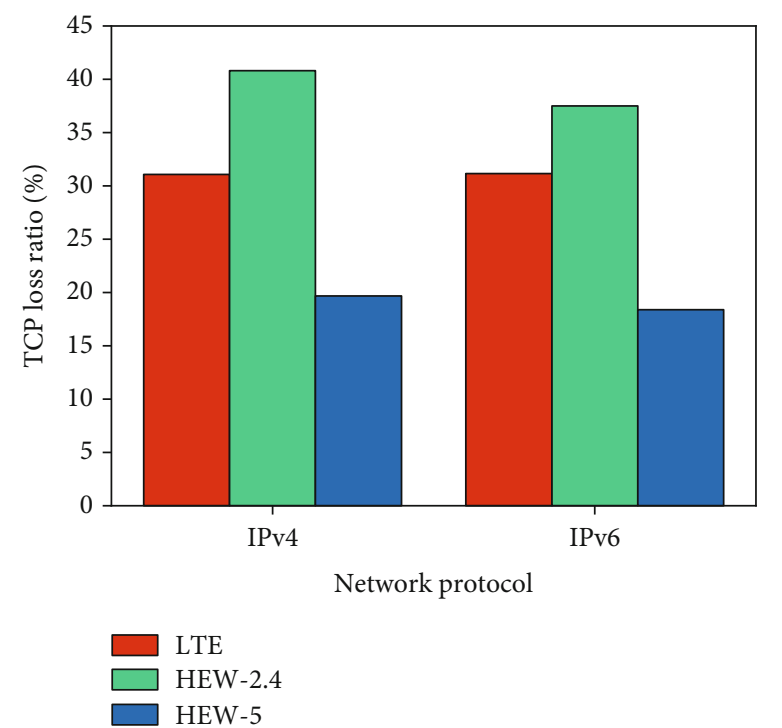

(c)

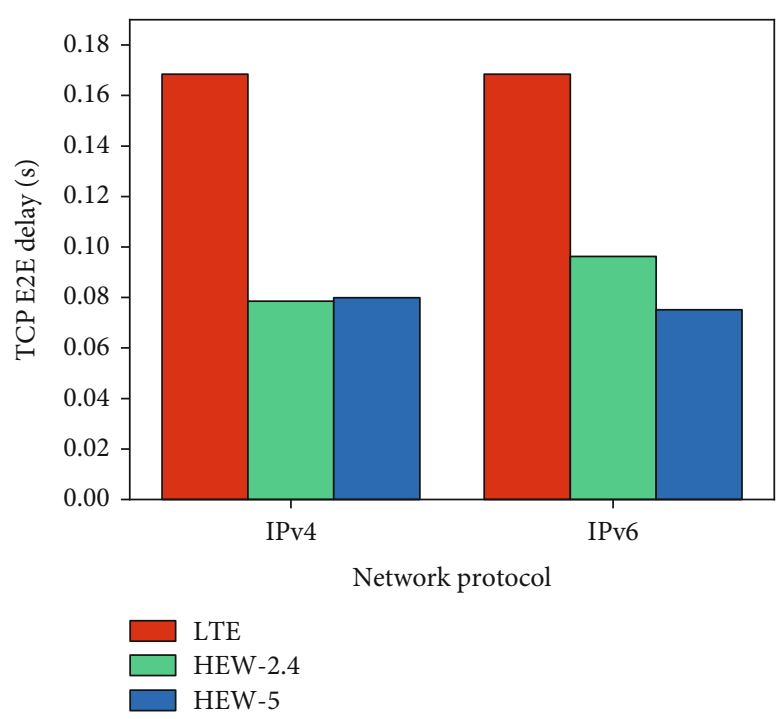

(b)

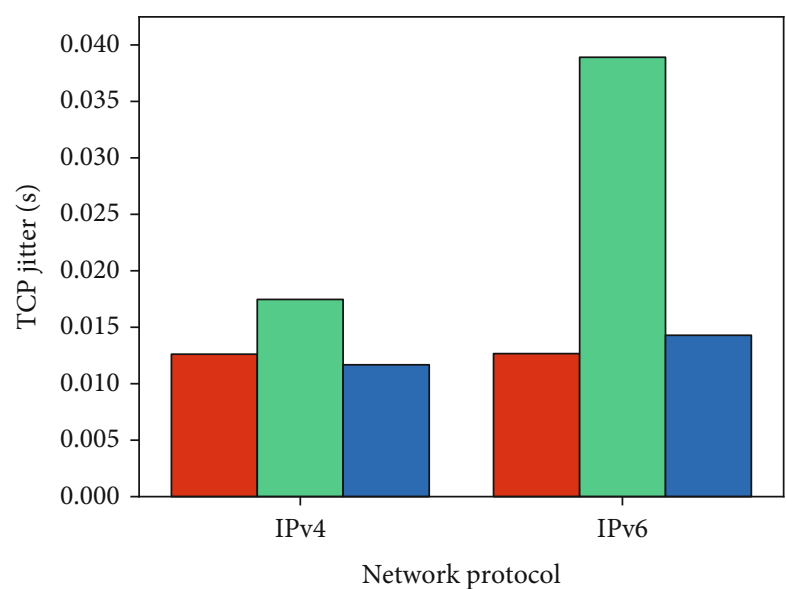

$\square$ LTE

FIgURE 20: Internet protocol efficiency: TCP (a) throughput, (b) delay, (c) loss ratio, and (d) jitter.

related to its operating in a lower frequency band. The IPv6 imposes higher overheads to the networks due to longer addresses. On the other hand, working at a lower frequency makes the HEW-2.4 slower than HEW-5. Thereby, the extra overheads of IPv6 can turn to bottleneck when the HEW-2.4 network is heavily overloaded by a large number of users all simultaneously exchanging bandwidth-intensive UDP applications such as video.

4.5. Payload Size Distribution. The significance of the influence imposed by different sizes of the data payload on the performance of the networks can differ. In this context, three different payload sizes including small, medium, and large (compared to the 1460 bytes as the MTU size) are exchanged over the LTE, HEW-2.4, and HEW-5 networks to determine the influence level and thereby identify the best suited size for these networks under high-density conditions. The results for error-sensitive data are presented in Figure 22.

The results indicate a very close and comparable performance of LTE and HEW-2.4 networks in contrast to a much better performance of the HEW-5 network. Based on the results, the transport payload size of error-sensitive applications as being small, medium, or large does not affect the performance of LTE, HEW-2.4, and HEW-5 networks under high-density conditions. Although a 1400B transport payload slightly improves the performance of the end users in the HEW-5 network in terms of the lower loss ratio and delay, the level of improvement is not considerable. These results are also consistent with the experimental testbed results for LTE in [44] and HEW in [42]. The reason that changing the payload size does not influence the performance of the LTE, HEW-2.4, and HEW-5 network is related 


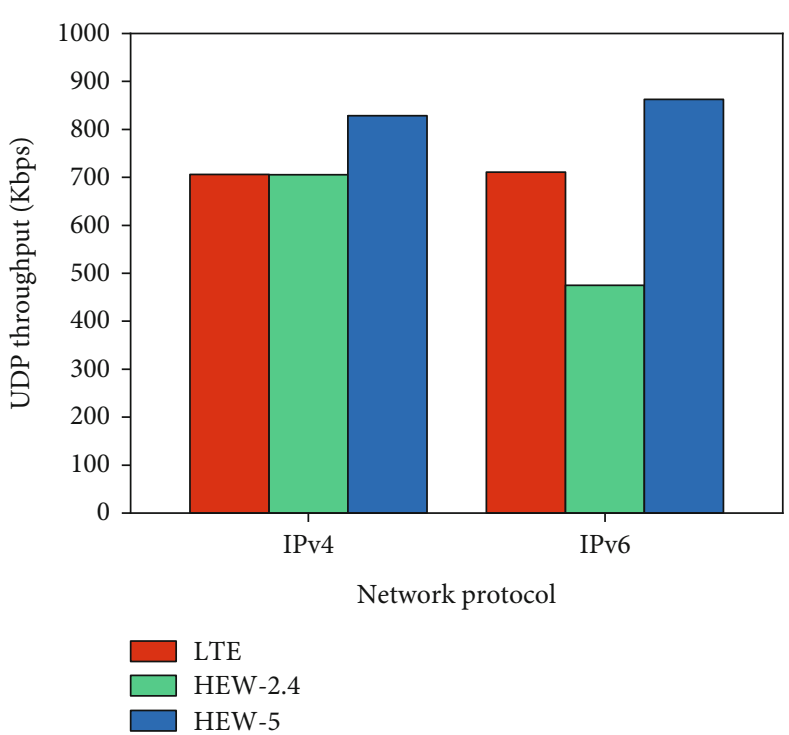

(a)

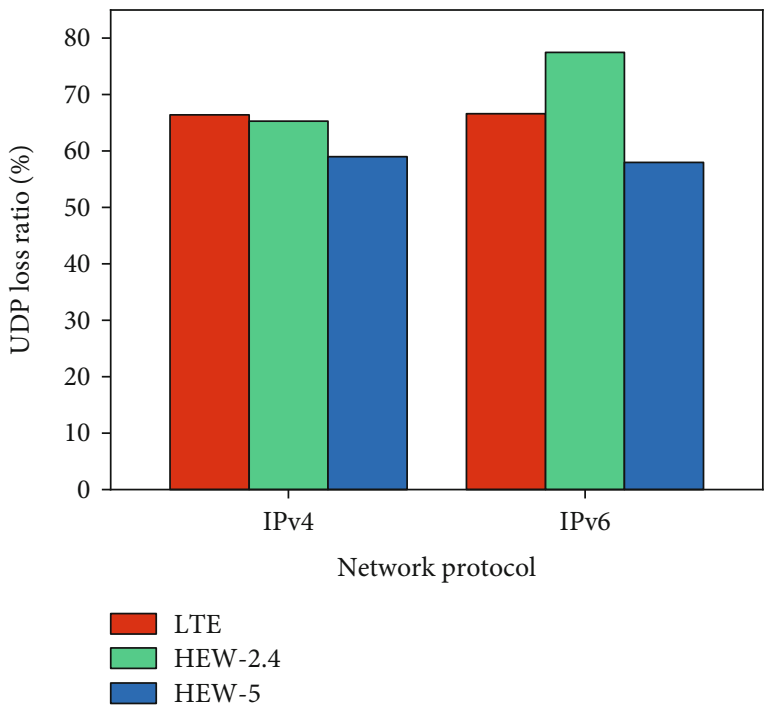

(c)

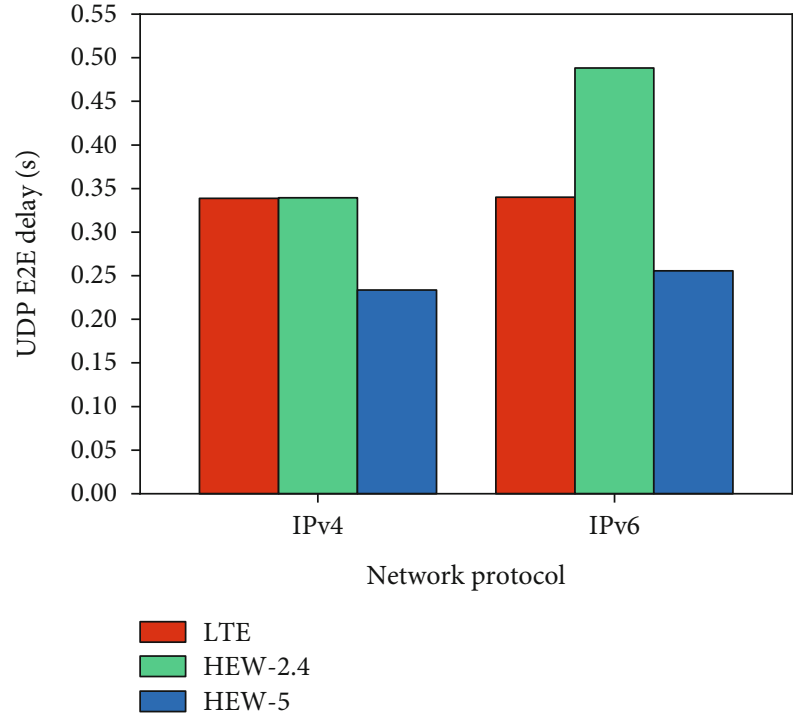

(b)

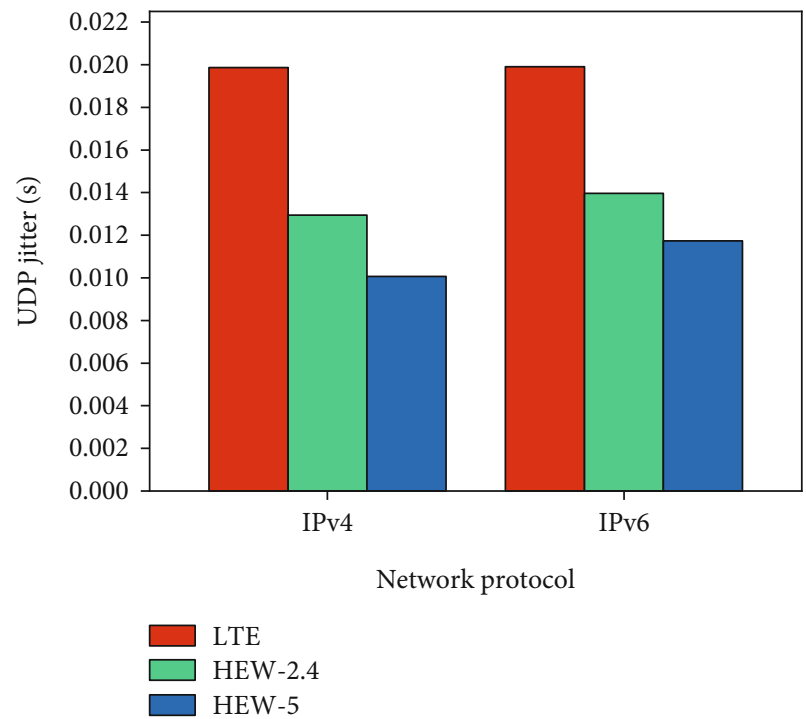

(d)

FIGURE 21: Internet protocol efficiency: UDP (a) throughput, (b) delay, (c) loss ratio, and (d) jitter.

to the fact that they reach their saturation limits by a large number of packet transmissions of the 100 active users at the same time. The very low throughput values confirm the saturation limit. The smaller packets mean lots of them, which will stress the network by doing more processing per unit of time. This issue gets even worst in dense environments with a large number of users all transmitting data at the same time. Further on this, smaller packets increase the overhead due to the additional headers. The larger packets, on the other hand, can solve these issues; still, at the same time, they reduce the bandwidth efficiency due to the segmentation overhead and also reduce the reliability as larger packets have a high probability of being corrupted during transmission. We also need to add TCP overheads including connection establishment, retransmission, and acknowledgment, to all these overheads. Thereby, the combined effects of all these overheads lead the three networks to their saturation limits. Furthermore, it is required to ensure whether the results differ in effect based on the type of applications that transmit different payload sizes in the networks. Thus, here, time-sensitive applications exchange data in high dense LTE, HEW-2.4, and HEW-5 networks to identify their performance differences. The results are provided in Figure 23.

The results show that, unlike error-sensitive data, the payload size of time-sensitive applications has a direct impact on the functionality of LTE, HEW-2.4, and HEW-5 networks under high-density conditions. The reason is the fact that the UDP does not include any TCP-related overhead as mentioned earlier. By removing the TCP overheads part, the networks are not as heavily loaded as they were before with the TCP data. The higher UDP throughput values compared to TCP confirm this. In terms of throughput, the highest and 


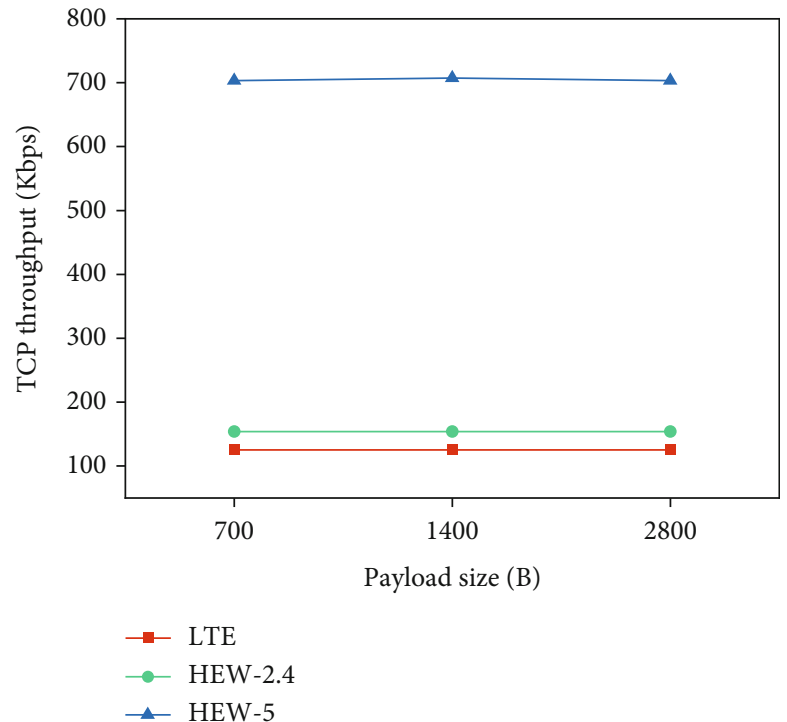

(a)

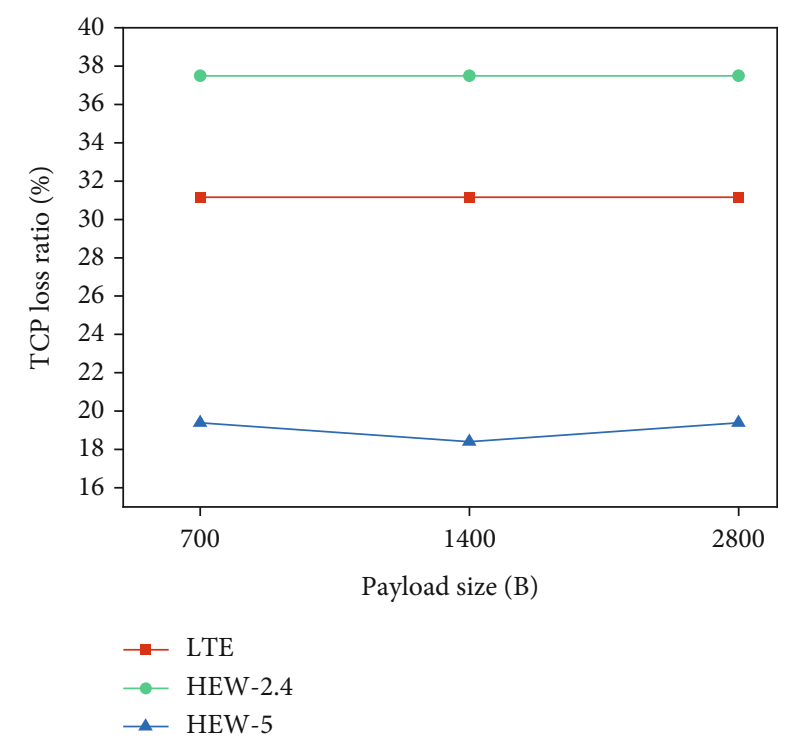

(c)

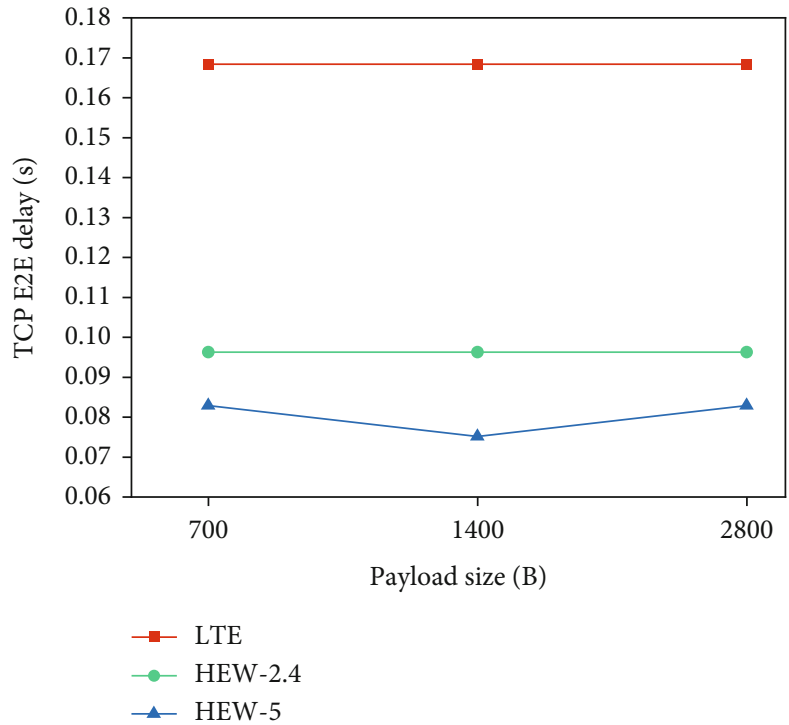

(b)

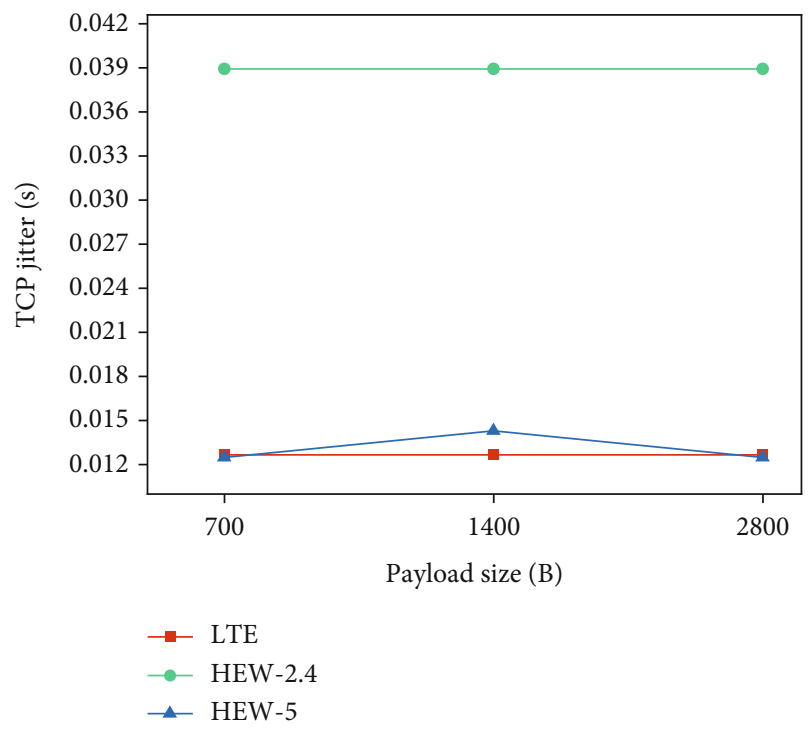

(d)

Figure 22: Payload size distribution: TCP (a) throughput, (b) delay, (c) loss ratio, and (d) jitter.

least values are obtained by HEW-5 and HEW-2.4 networks, respectively. In this context, small (700B) and medium (1400B) payloads do not affect the end users in LTE and HEW-5 networks, whereas medium payloads improve the performance of the HEW-2.4 network. With regard to larger payloads (2800B), we observe that going beyond the MTU size will significantly degrade the performance of LTE, HEW-2.4, and HEW-5 in high-density environments. In terms of delay, we observe a slight reduction when larger payloads (2800B) are exchanged in high dense LTE, HEW-2.4, and HEW-5 networks, although the reduction level is not considerable and does not efficiently affect the timesensitive data. Based on jitter results, the HEW-5 network provides similar values for its end users regardless of the payload size which is in contrast to LTE and HEW-2.4 networks, where larger payloads considerably increase the jitter which is an essential parameter for time-sensitive applications. On this basis, when jitter is a matter of importance in the network, using smaller payloads is more efficient. Additionally, the loss ratio results imply that to have the least ratio, using $1400 \mathrm{~B}$ payloads is the right option under dense conditions of LTE, HEW-2.4, and HEW-5 deployments.

\section{Conclusion}

This work proposes a model to enhance the capacity of high dense LTE, HEW-2.4, and HEW-5 networks through the proper selection of the six deployment options. Regarding the operating channel management, the results show better performance of LTE with wider channels. Although this conclusion is applicable for HEW-2.4 and HEW-5 networks, the $160 \mathrm{MHz}$ channel is the exception. In this context, 


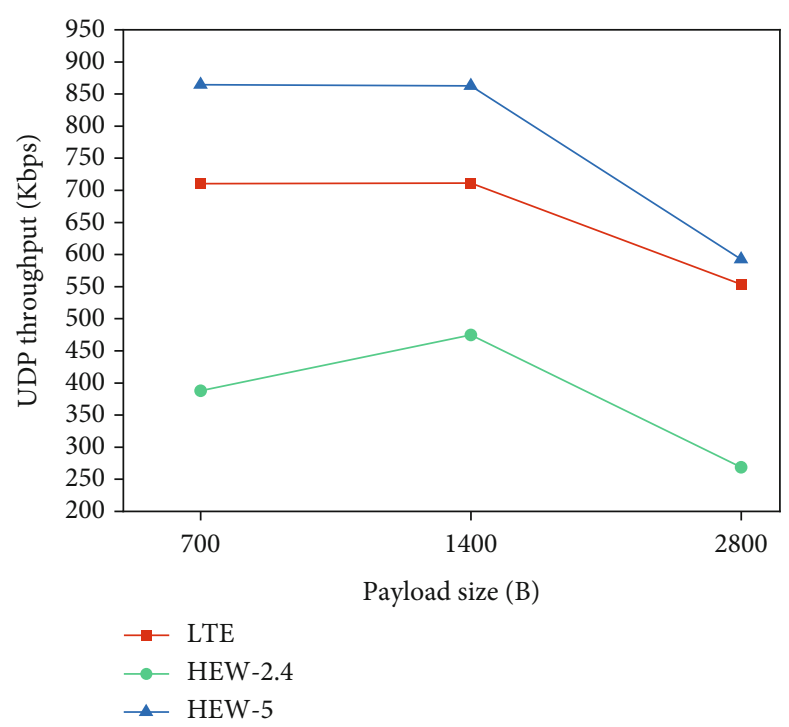

(a)

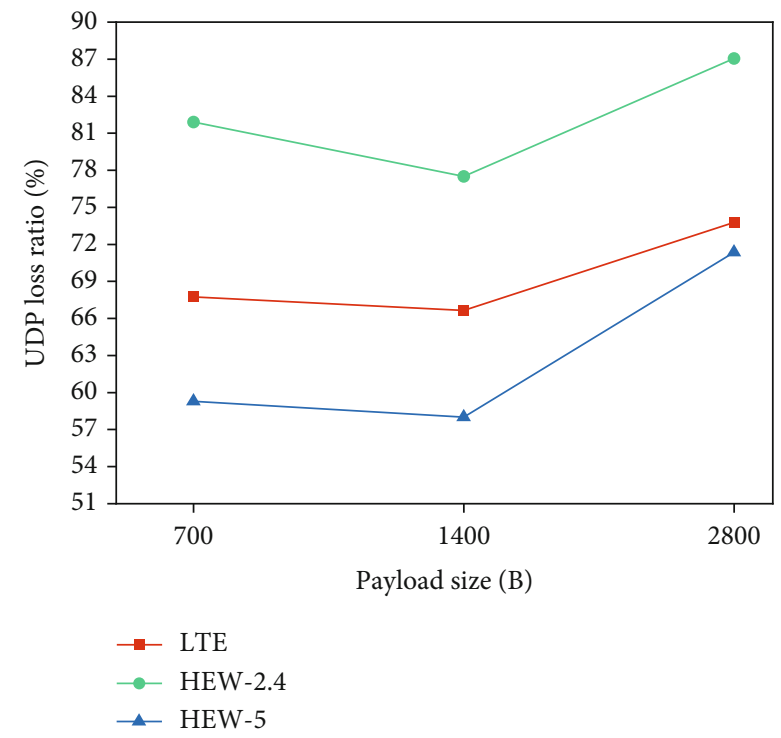

(c)

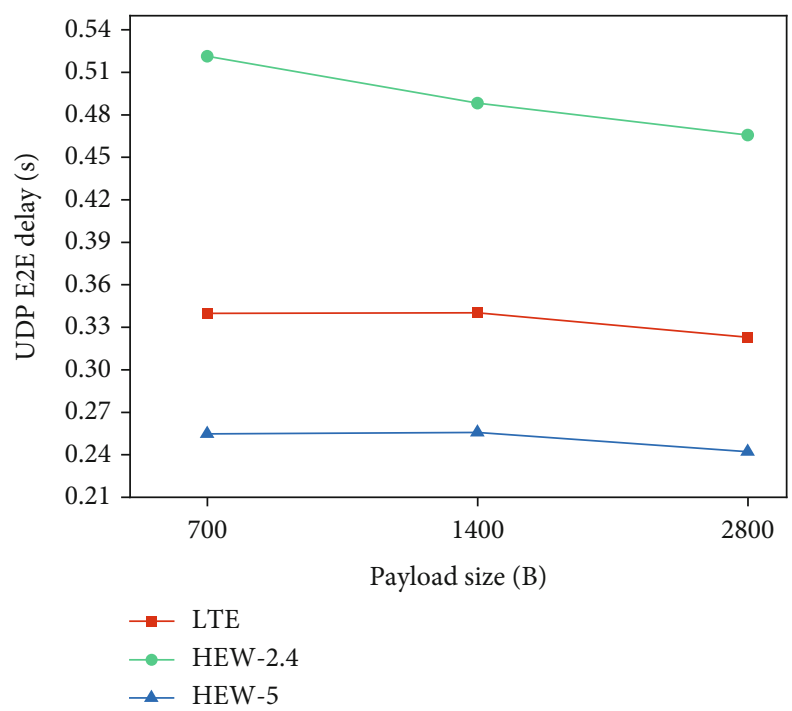

(b)

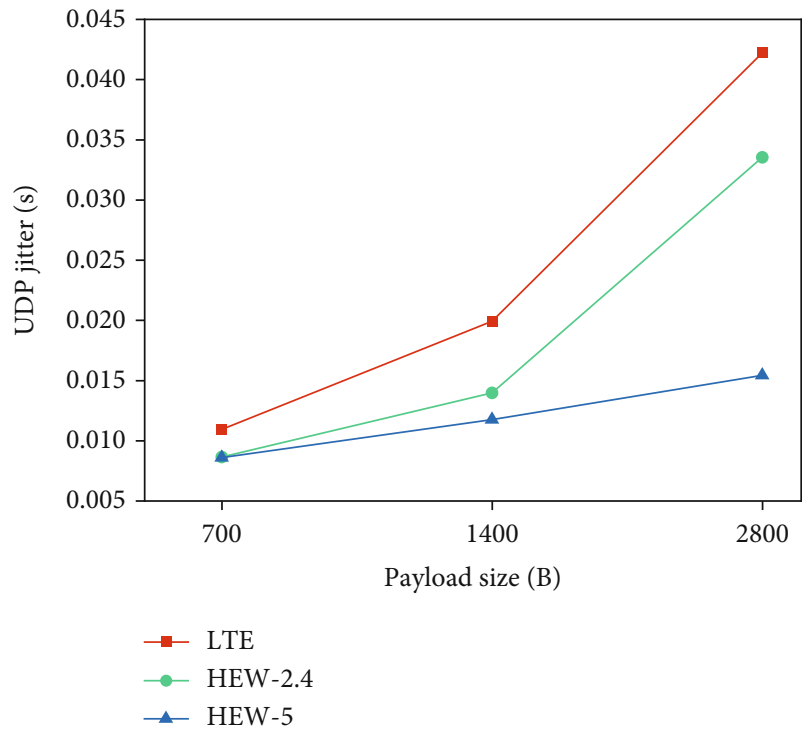

(d)

Figure 23: Payload size distribution: UDP (a) throughput, (b) delay, (c) loss ratio, and (d) jitter.

considering that the wider channels are subject to more interference, and also considering the close performance of 40 and $80 \mathrm{MHz}$ channels, using $40 \mathrm{MHz}$ will be a better choice in high-density areas. With reference to the maximum connection limit, as the networks get denser with more users, the performance reduces so that the LTE network is more subject to fail its users than HEW-2.4 and HEW-5, particularly in the presence of error-sensitive applications. For error-sensitive applications, 30 and 70 are the connection limits for LTE and HEW networks, respectively, while for time-sensitive applications, the limit is nearly 90 for all three networks. Regarding the antenna configuration, while spatial multiplexing mode is the best suited for the LTE network for enhancing the overall network capacity, the four available modes for HEW-2.4 and HEW-5 networks contribute equivalently. In connection with the internet protocol efficiency,
IPv6 can achieve close performance in LTE and HEW networks compared to IPv4 under high-density conditions. In this context, although IPv4 appears to be more efficient for HEW-2.4, the difference is not considerable. Thus, looking at the future and considering the growing demand for more IP spaces, using IPv6 is a better option. Regarding the payload size distribution, the performance of error-sensitive applications does not get affected by the size. On the contrary, the best suited payload size for the time-sensitive applications is under the MTU size.

\section{Data Availability}

The data sharing can be available upon request to the corresponding author. 


\section{Conflicts of Interest}

The author declares that there is no conflict of interest regarding the publication of this paper.

\section{References}

[1] J. So and J. Lee, "A Simple and Practical Scheme Using Multiple Channels for Improving System Spectral Efficiency of Highly Dense Wireless LANs," Hindawi Wireless Communications and Mobile Computing, vol. 2019, article 2149606, pp. 117, 2019.

[2] Cisco, "High Density Wi-Fi Deployments. Resource document," 2020, https://documentation.meraki.com/ Architectures_and_Best_Practices/Cisco_Meraki_Best_ Practice_Design/Best_Practice_Design_-_MR_Wireless/ High_Density_Wi-Fi_Deployments.

[3] S. A. Nora, R. Alubadya, and W. A. Kamilc, "Simulated Performance of TCP, SCTP, DCCP and UDP Protocols over 4G Network," in 8th International Conference on Advances in Information Technology (IAIT2016), Macau, China, 2016.

[4] L. Giupponia, T. Hendersonb, B. Bojovica, and M. Miozzoa, "Simulating LTE and Wi-Fi coexistence in unlicensed spectrum with ns-3," 2016, https://arxiv.org/abs/1604.06826, Networking and Internet Architecture.

[5] D. J. Deng, S. Y. Lien, J. Lee, and K. C. Chen, "On quality-ofservice provisioning in IEEE 802.11ax WLANs," IEEE Access, vol. 4, pp. 6086-6104, 2016.

[6] Y. Nagai, L. Zhang, T. Okamawari, and T. Fujii, "Delay Performance Analysis of LTE in Various Traffic Patterns and Radio Propagation Environments," in IEEE 77th Vehicular Technology Conference (VTC Spring), Dresden, Germany, 2013.

[7] J. Hyun, J. Li, H. Kim, J. H. Yoo, and J. W. K. Hong, "IPv4 and IPv6 Performance Comparison in IPv6 LTE Network," in 2015 17th Asia-Pacific Network Operations and Management Symposium (APNOMS), Busan, South Korea, 2015.

[8] P. Shah, K. Sakhardande, and G. Shah, "Performance Analysis of LTE Network Using QAM and MIMO Configuration," in 2018 IEEE International Students' Conference on Electrical, Electronics and Computer Science (SCEECS), Bhopal, India, 2018.

[9] H. Hafez and A. Mokhtar, "Performance Analysis of Transmit Diversity and Spatial Multiplexing MIMO Techniques in LTE Cell Edge Coverage Areas," in ICSIE '19: 2019 8th International Conference on Software and Information Engineering, Cairo, Egypt, 2019.

[10] B. Bellalta and K. K. Szott, "AP-initiated Multi-User Transmissions in IEEE 802.11ax WLANs," Elsevier Ad Hoc Networks, vol. 85, 2019.

[11] J. S. S. Mahecha, S. Cespedes, and J. B. Jimenez, "QoS evaluation of the future high-efficiency IEEE 802.11ax WLAN standard," in 2018 IEEE Colombian Conference on Communications and Computing (COLCOM), Medellin, Colombia, 2018.

[12] A. K. Ajami and H. Artail, "Analyzing the impact of the coexistence with IEEE 802.11ax Wi-Fi on the performance of DSRC using stochastic geometry modeling," IEEE transactions on communications, vol. 67, no. 9, pp. 6343-6359, 2019.

[13] O. Sharon and Y. Alpert, "Advanced IEEE 802.11ax TCP aware scheduling under unreliable channels," International Journal of Communication Systems, vol. 32, no. 14, 2019.

[14] D. Bankov, A. Didenko, E. Khorvo, and A. Lyakhov, "OFDMA uplink scheduling in IEEE 802.11ax networks," in 2018 IEEE
International Conference on Communications (ICC), Kansas City, MO, USA, 2018.

[15] G. Naik, S. Bhattarai, and J. M. Park, "Performance analysis of uplink multi-user OFDMA in IEEE 802.11ax," in 2018 IEEE International Conference on Communications (ICC), Kansas City, MO, USA, 2018.

[16] L. Kiwoli, A. Sam, and E. Manasseh, "Performance analysis of carrier aggregation for various mobile network implementations scenario based on spectrum allocated," International Journal of Wireless \& Mobile Networks, vol. 9, no. 5, pp. 41-53, 2017.

[17] S. A. A. Termizi, S. Rahmat, and M. A. Qadeer, "LTE-U over Wi-Fi," in IEEE Thirteenth International Conference on Wireless and Optical Communications Networks (WOCN), Hyderabad, India, 2016.

[18] P. Gahan, D. Standeford, M. Sims, C. Viola, and J. Watson, "4G and 5G Spectrum Guide," Policy Tracker, 2019.

[19] A. Hameed, L. F. Abdulrazak, Z. A. Aljawary, and F. L. Malallah, "Link-to-system MIMO interference analysis for LTE coexistence in $2.6 \mathrm{GHz}$ frequency band," International Journal of Applied Engineering Research, vol. 12, no. 15, pp. 5226-5233, 2017.

[20] The 253rd Generation Partnership Project, "3GPP TS 38.101-1 V16.0.0 (2019-06)," Technical Specification Group Radio Access Network, User Equipment (UE) radio transmission and reception, Part 1: Range 1 Standalone (Release 16), 2019.

[21] The 3rd Generation Partnership Project, "3GPP TS 36.508 V13.0.1," Evolved Universal Terrestrial Radio Access (E-UTRA) and Evolved Packet Core (EPC), Common test environments for User Equipment (UE) conformance testing (Release 13), 2016.

[22] D. J. Deng, Y. P. Lin, X. Yang et al., "IEEE 802.11ax: highly efficient WLANs for intelligent information infrastructure," IEEE Communications Magazine, vol. 55, no. 12, pp. 52-59, 2017.

[23] P. Sharda, H. Singh, and A. Sheetal, "Optimisation of LTE system with open- and closed-loop spatial multiplexing transmission modes," Australian Journal of Electrical and Electronics Engineering, vol. 14, no. 3-4, pp. 88-92, 2018.

[24] M. Ammar, B. Nsiri, W. Hakimi, and M. Eljamai, "A comprehensive study of open-loop spatial multiplexing and transmit diversity for downlink LTE," International Journal of Computer Science and Telecommunications, vol. 5, no. 2, 2014.

[25] A. S. Konstantinov and A. V. Pestryakov, "Analysis of the Transmission Modes and Downlink Control Information in the LTE-Advanced/5G Network," in 2019 Systems of Signals Generating and Processing in the Field of on Board Communications, Moscow, Russia, 2019.

[26] G. Alvarez, M. Alvarez, F. Bagalciague et al., "Multi-client emulation platform for performance testing of high density 802.11 networks," in IEEE Wireless Days (WD), Toulouse, France, 2016.

[27] I. Coonjah, P. C. Catherine, and K. M. S. Soyjaudah, "Experimental Performance Comparison between TCP Vs UDP Tunnel Using OpenVPN," in 2015 International Conference on Computing, Communication and Security (ICCCS), Pamplemousses, Mauritius, 2016.

[28] I. Maslouhi, E. Miloud, K. Ghoumid, and K. Baibai, “Analysis of end-to-end packet delay for Internet of Things in wireless communications," International Journal of Advanced Computer Science and Applications, vol. 9, no. 9, 2018.

[29] M. Polese, F. Chiariotti, E. Bonetto, F. Rigotto, A. Zanella, and M. Zorzi, "A survey on recent advances in transport layer 
protocols," IEEE Communications Surveys \& Tutorials, vol. 21, no. 4, pp. 3584-3608, 2019.

[30] A. Larmo, A. Ratilainen, and J. Saarinen, "Impact of CoAP and MQTT on NB-IoT system performance," Journal of Sensors, vol. 19, no. 1, 2019.

[31] W. Simpson, Video Over IP: A Practical Guide to Technology and Applications, Elsevier, 2006.

[32] M. al-Medhwahi, F. Hashim, B. Ali, and A. Sali, "Impact of packet size in adaptive cognitive radio sensor network," Wireless Communications and Mobile Computing, vol. 2018, Article ID 3051204, 9 pages, 2018.

[33] S. K. Apeksha and M. S. Usha, "An overview of RLC layer in 4G LTE," International Journal of Engineering Development and Research, vol. 3, no. 2, 2015.

[34] Z. Huang, L. Y. Por, T. F. Ang, M. H. Anisi, and M. S. Adam, "Improving the accuracy rate of link quality estimation using fuzzy logic in Mobile wireless sensor network," Advances in Fuzzy Systems, vol. 2019, Article ID 3478027, 13 pages, 2019.

[35] F. Afroz, R. Subramanian, R. Heidary, K. Sandrasegaran, and S. Ahmed, "SINR, RSRP, RSSI and RSRQ measurements in long term evolution networks," International Journal of Wireless \& Mobile Networks, vol. 7, no. 4, pp. 113-123, 2015.

[36] R. Nofitasari and S. Haryadi, "Self-healing mechanism with RSRP measurement in LTE network," in 2017 3rd International Conference on Wireless and Telematics (ICWT), Palembang, Indonesia, 2017.

[37] A. K. Sarangi and A. Datta, "Capacity Comparison of SISO, SIMO, MISO \& MIMO Systems," in 2018 Second International Conference on Computing Methodologies and Communication (ICCMC), Erode, India, 2018.

[38] I. A. Mejibli and S. A. Majeed, "Challenges of Using MIMO Channel Technology in 5G Wireless Communication Systems," in Majan International Conference (MIC), Muscat, Oman, 2018.

[39] O. Rioul and J. C. Magossi, "On Shannon's formula and Hartley's rule: beyond the mathematical coincidence," Entropy, vol. 16, no. 9, pp. 4892-4910, 2014.

[40] M. S. Gast, 802.11ac: a survival guide: Wi-fi at gigabit and beyond, O'Reilly Media Inc. Publications, 2013.

[41] “Technical specification,” LTE; Evolved Universal Terrestrial Radio Access (E-UTRA); Physical layer procedures (3GPP TS 36.213 version 14.2.0 Release 14), 2017.

[42] S. Muhammad, J. Zhao, and H. H. Refai, "An empirical analysis of IEEE 802.11ax," in International Conference on Communications, Signal Processing and their Applications, Sharjah, UAE, 2020.

[43] H. R. Chayon, K. Dimyati, H. Ramiah, and A. W. Reza, “An improved radio resource management with carrier aggregation in LTE advanced," Applied Sciences, vol. 7, no. 4, p. 394, 2017.

[44] M. Malekzadeh, "IPv6 transition measurements in LTE and VHT Wi-Fi mobile networks," IEEE Access, vol. 7, pp. 183024-183039, 2019.

[45] F. Li, X. Wang, T. Pan, and J. Yang, "A Case Study of IPv6 Network Performance: Packet Delay, Loss, and Reordering," Mathematical Problems in Engineering, vol. 2017, Article ID 3056475, 10 pages, 2017.

[46] T. Pattanasophon, S. Thanee, S. Limpayaraya, and T. Kaewkiriya, "A study on performance of IPv4, IPv6 on wireless network (IEEE 802.11ac) with wireless security protocols (WEP, WPA and WPA2)," TNI Journal of Engineering and Technology, vol. 5, no. 1, 2017. 